\title{
Preliminary Analysis of Aircraft Loss of Control Accidents: Worst Case Precursor Combinations and Temporal Sequencing
}

\author{
Christine M. Belcastro* \\ NASA Langley Research Center \\ Hampton, Virginia, 23681 \\ Richard L. Newman ${ }^{\ddagger}$ \\ Crew Systems \\ Seattle, Washington, 98165 \\ Dennis A. Crider ${ }^{* *}$ \\ National Transportation Safety Board \\ Washington D.C., 20594
}

\author{
Loren Groff ${ }^{\dagger}$ \\ National Transportation Safety Board \\ Washington D.C., 20594 \\ John V. Foster ${ }^{\S}$ \\ NASA Langley Research Center \\ Hampton, Virginia, 23681 \\ David H. Klyde ${ }^{\dagger \dagger}$ \\ Systems Technology, Inc. \\ Hawthorne, CA, 902504
}

\author{
and \\ A. McCall Huston \\ Massachusetts Institute of Technology, Cambridge, MA 02139
}

\begin{abstract}
Aircraft loss of control (LOC) is a leading cause of fatal accidents across all transport airplane and operational classes, and can result from a wide spectrum of hazards, often occurring in combination. Technologies developed for LOC prevention and recovery must therefore be effective under a wide variety of conditions and uncertainties, including multiple hazards, and their validation must provide a means of assessing system effectiveness and coverage of these hazards. This requires the definition of a comprehensive set of LOC test scenarios based on accident and incident data as well as future risks. This paper defines a comprehensive set of accidents and incidents over a recent $\mathbf{1 5}$ year period, and presents preliminary analysis results to identify worst-case combinations of causal and contributing factors (i.e., accident precursors) and how they sequence in time. Such analyses can provide insight in developing effective solutions for LOC, and form the basis for developing test scenarios that can be used in evaluating them. Preliminary findings based on the results of this paper indicate that system failures or malfunctions, crew actions or inactions, vehicle impairment conditions, and vehicle upsets contributed the most to accidents and fatalities, followed by inclement weather or atmospheric disturbances and poor visibility. Follow-on research will include finalizing the analysis through a team consensus process, defining future risks, and developing a comprehensive set of test scenarios with correlation to the accidents, incidents, and future risks. Since enhanced engineering simulations are required for batch and piloted evaluations under realistic LOC precursor conditions, these test scenarios can also serve as a high-level requirement for defining the engineering simulation enhancements needed for generating them.
\end{abstract}

\footnotetext{
* Senior Researcher, Dynamic Systems and Control Branch, MS 308, E-Mail: christine.m.belcastro@nasa.gov; AIAA Associate Fellow.

† National Resource Specialist, Safety Data Systems and Analysis, RE-10, E-Mail: groffl@ntsb.gov.

${ }^{\ddagger}$ Retired, FAA, Post Office Box 25054, E-Mail: dicknewman@earthlink.net; AIAA Associate Fellow.

$\S$ Senior Researcher, Flight Dynamics Branch, MS 308, E-Mail: john.v.foster@nasa.gov; AIAA Associate Fellow.

** Chief Technical Advisor, Vehicle Simulation, RE-1, E-Mail: criderd@ntsb.gov; AIAA Senior Member.

t† Technical Director, E-Mail: dklyde@systemstech.com; AIAA Associate Fellow.

㧊 Engineering undergraduate student, E-Mail: mhuston@mit.edu.
} 


\section{Nomenclature}

$\begin{array}{ll}\text { CAST } & =\text { Commercial Aviation Safety Team } \\ \text { EASA } & =\text { European Aviation Safety Agency } \\ F A A & =\text { Federal Aviation Administration } \\ \text { ICAO } & =\text { International Civil Aviation Organization } \\ \text { LOC } & =\text { Loss of Control (in-flight) } \\ \text { NASA } & =\text { National Aeronautics and Space Administration } \\ \text { NTSB } & =\text { National Transportation Safety Board }\end{array}$

\section{Introduction}

ircraft loss of control (LOC) is a leading cause of fatal accidents across all transport airplane and operational classes. ${ }^{1,2,3}$ LOC can be described as motion that is:

- $\quad$ outside the normal operating flight envelopes; not predictably altered by routine pilot control inputs;

- characterized by nonlinear effects, such as kinematic/inertial coupling;

- $\quad$ disproportionately large responses to small state variable changes, or oscillatory/divergent behavior;

- likely to result in high angular rates and displacements; and

- $\quad$ characterized by the inability to maintain heading, altitude, and wings-level flight. ${ }^{4}$

LOC is therefore fundamentally a dynamics and control problem, but there are many causal and contributing factors (or precursors) that can lead to LOC. ${ }^{5,6,7}$ The primary causes include: entry into a vehicle upset condition; reduction or loss of control effectiveness; changes to the vehicle dynamic response in relation to handling/flying qualities; and combinations of these. There are numerous factors that have historically led or contributed to LOC. These can be grouped into three major categories: adverse onboard conditions, external hazards and disturbances, and abnormal vehicle dynamics and upset conditions. LOC causal and contributing factors within these categories are summarized in Fig. 1. Adverse onboard conditions include vehicle problems (i.e., vehicle impairment, vehicle damage, or system failures) and inappropriate crew actions or inaction. External hazards and disturbances consist of inclement weather conditions, atmospheric disturbances, poor visibility, and obstacles (fixed and moving) that require abrupt maneuvering for avoidance. Examples of abnormal vehicle dynamics include oscillatory response, uncommanded motions, and non-intuitive control response. Upset conditions include a variety of off-nominal or extreme flight conditions and abnormal trajectories (e.g., abnormal attitude, uncontrolled descent, and stall / departure). The complexity of LOC is clearly illustrated in Fig. 1, particularly considering that many LOC accidents involve combinations of the causal and contributing factors that are listed.

Solutions for LOC must therefore be developed to provide prevention and recovery capabilities under a wide variety of hazards (and their combinations) that can lead to LOC. ${ }^{8,9}$ One onboard system concept for accomplishing this is illustrated in Fig. 2. The colors depicted in Fig. 2 are representative of the following functions: vehicle health state detection capabilities are indicated by green, vehicle flight safety state assessment and resilient guidance and control capabilities are shown in blue, crew-system interface information and support capabilities are shown in yellow, and onboard modeling capabilities are shaded in purple. The signals depicted in Fig. 2 represent vector quantities and are defined as follows: " $\mathrm{x}$ " is the vehicle state, " $y$ " represents measurable outputs, " $\mathrm{z}$ " represents controlled variables (which can be mode-dependent), "u" represents control inputs (with subscript "p" denoting pilot input commands, and subscript " $c$ " denoting control system commands), " $\mathrm{n}$ " represents noise signals, " $\mathrm{f}$ " represents failures (and in the case of jammed actuators, for example, can represent persistent asymmetric forces acting on the aircraft), and "d" represents external disturbances. These technologies may also be aimed at specific precursors that are shown to cause a significant proportion of accidents.

The validation of technologies developed for LOC prevention and recovery, such as those illustrated in Fig. 2, poses significant challenges. All LOC hazards and their combinations cannot be fully replicated in piloted simulation or flight test evaluations. However, the validation process must provide some measure of assurance that the new vehicle safety technologies are effective and that they do no harm - i.e., that they themselves do not introduce new safety risks. Moreover, a means of assessing hazards coverage must also be included in the validation framework. 


\begin{tabular}{|c|c|c|}
\hline & & $\frac{\text { Causal \& }}{\text { Contributing Factors }}$ \\
\hline $\begin{array}{l}\text { Generally, LOC is described } \\
\text { as motion that is: } \\
\text { - outside normal envelopes } \\
\text { - not predictably altered by } \\
\text { pilot control inputs } \\
\text { - characterized by nonlinear } \\
\text { effects, } \\
\text { - disproportionately large } \\
\text { responses to small state } \\
\text { variable changes, } \\
\text { - oscillatory/divergent behavior } \\
\text { - likely to result in high angular } \\
\text { rates / displacements, } \\
\text { - characterized by the inability to } \\
\text { maintain heading, altitude, and } \\
\text { wings-level flight }\end{array}$ & $\begin{array}{l}\text { Primary } \\
\begin{array}{l}\text { Causes } \\
\text { 1. Entry into vehicle } \\
\text { upset condition } \\
\text { (e.g., Stall) }\end{array} \\
\text { 2. Reduction or loss } \\
\text { of control } \\
\text { effectiveness } \\
\text { 3. Changes to vehicle } \\
\text { dynamic response } \\
\text { and handling / } \\
\text { flying qualities }\end{array}$ & $\begin{array}{l}\text { - Adverse onboard conditions: } \\
\text { - vehicle impairment } \\
\text { » Inappropriate vehicle configuration, } \\
\text { contaminated airfoil, improper loading, vehicle } \\
\text { damage to airframe and engines } \\
\text { - system faults, failures, and errors } \\
\text { » Control component, engine, sensor system, flight } \\
\text { deck instrumentation, non-control component } \\
\text {-inappropriate crew action / inaction } \\
\text { » Loss of aircraft attitude, energy, or system state } \\
\text { awareness, aggressive maneuver, abnormal } \\
\text { control input, ineffective recovery, improper } \\
\text { procedure, crew fatigue / impairment } \\
\text { - External hazards and disturbances: } \\
\text { - inclement weather \& atmospheric disturbances } \\
\text { » wind shear, turbulence, rain / thunderstorms, } \\
\text { snow / icing, wake vortices } \\
\text { - poor visibility (fog / haze, night) } \\
\text { - obstacle (fixed or moving) } \\
\text { - Abnormal dynamics \& vehicle upsets: } \\
\text { - abnormal vehicle dynamics \& control response } \\
\text { - abnormal attitude, airspeed, angular rates, } \\
\text { asymmetric forces, or flight trajectory } \\
\text { - uncontrolled descent (including spiral dive) } \\
\text { - stall/departure from controlled flight }\end{array}$ \\
\hline
\end{tabular}

Figure 1. LOC key characteristics, primary causes, and causal \& contributing factors.

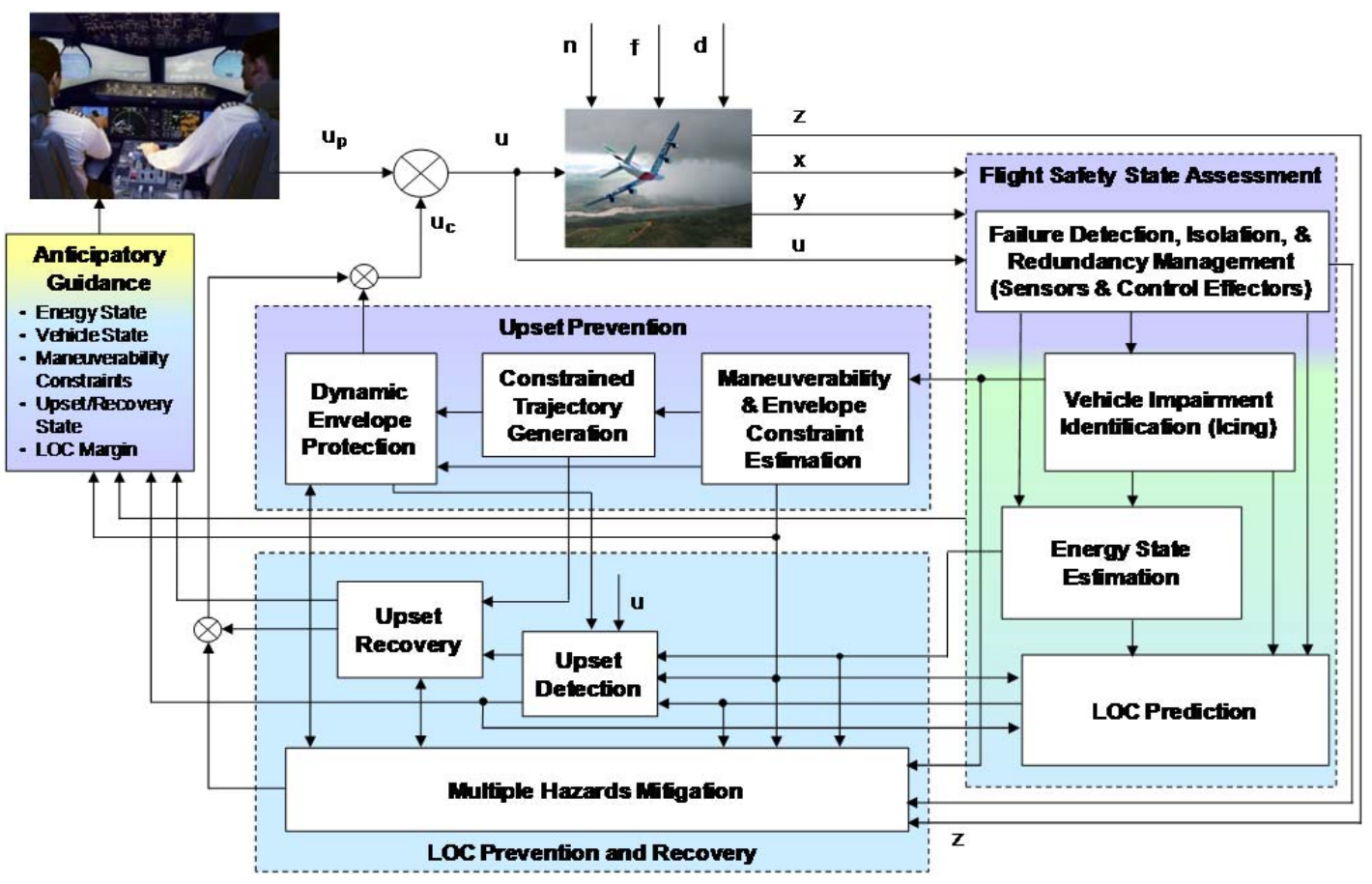

Figure 2. Onboard system concept for LOC prevention and recovery. 
A validation framework involving analysis, simulation, and experimental testing was previously developed for safety-critical integrated systems operating under hazardous conditions that can lead to LOC, ${ }^{10,11}$ and a preliminary set of LOC test scenarios ${ }^{12}$ was developed based on a limited accident set defined over a thirty year time period. The objectives of the current research are to define an extensive accident (and incident) set over a recent fifteen year time period, perform a thorough analysis of this accident / incident set based on a team consensus process, and develop a comprehensive set of test scenarios based on this analysis and an identified set of potential future risks. This paper presents preliminary results of this research. Specifically, this paper presents a set of 275 LOC accidents and incidents from 1996-2010, and preliminary analysis results to identify worst-case combinations of causal and contributing factors and how they sequence in time. Final analysis results and the set of LOC test scenarios, both based on a team consensus process, will be published separately. The test scenarios will be based on the analysis of accidents, incidents, and future risks, and will be developed for use in the validation of onboard systems technologies for LOC prevention and recovery. Since enhanced engineering simulations are required for batch and piloted evaluations under realistic LOC precursor conditions, these test scenarios can also serve as a high-level requirement for defining the simulation enhancements needed for generating realistic LOC test scenarios.

Section II defines the accident / incident set used in the analysis, and presents preliminary analysis results in terms of worst-case hazards combinations and how they sequence in time. Section III discusses a preliminary set of future potential risks that are relevant to LOC. Section IV discusses follow-on work, which includes finalizing the accident analysis results, finalizing the set of future risks, and developing a comprehensive set of LOC test scenarios based on the final accident / incident analysis and future risks. Section V provides a summary of the results of this paper and some concluding remarks. Appendix A provides the full set of accidents and incidents used in the analysis of Section II, and Appendix B presents LOC sequence diagrams resulting from the analysis.

\title{
II. Aircraft Loss-of-Control Accident / Incident Set and Preliminary Analysis
}

This section presents a detailed analysis of aircraft accidents and incidents (to be equivalently referred to as "events" in this paper). The primary accident / incident set will be categorized as LOC, but LOC-related accidents (e.g., resulting from control component failures and/or vehicle damage sufficient to alter vehicle dynamics and control characteristics) were also evaluated.

\section{A. Accident / Incident Set Definition}

Transport airplane loss-of-control events were reviewed for the fifteen year period 1996 through 2010. Only airplanes certified under Transport Category ${ }^{*}$ or Commuter Category ${ }^{\dagger}$ were included. Only normal commercial or non-revenue flights were included, such as scheduled or non-scheduled passenger or cargo flights, positioning flights, or executive flights. Events that occurred during demonstration, military, training, or test flights were not considered, nor were owner-flown business jet operations.

Accident databases were searched using the terms: "loss-of-control," "upset," “unusual attitude," "stall," and "uncontrolled." * The following databases were searched:

- $\quad$ Aircraft Accident Report DVD ${ }^{13}$

- Australian Transport Safety Bureau (ATSB)

- Aviation Safety Network (ASN)

\begin{abstract}
${ }^{*}$ Transport Category airplanes are certified under the provisions of Federal Aviation Regulations Part 25 or EASA Certification Standards Part 25 or predecessor regulations.

${ }^{\dagger}$ Commuter Category airplanes are certified under the provisions of Federal Aviation Regulations Part 23 or EASA Certification Standards Part 23 or predecessor regulations. Commuter Category airplanes are limited to propeller airplanes with maximum capacity of 19 passengers and maximum approved takeoff weights of 19,000 lb.

${ }^{\ddagger}$ The selection criteria used in this analysis resulted in a broader range of events than classification schemes like the CAST / ICAO Common Taxonomy Team occurrence category LOC-I (see: http://intlaviationstandards.org/CommonTaxonomies.html) and included accidents and incidents in which the flight crew failed to maintain aircraft control, as well as events involving abrupt maneuvers, weather encounters, and reduced control capability due to equipment malfunction or failure.
\end{abstract}

4

American Institute of Aeronautics and Astronautics 
- Canadian Transportation Safety Board (TSB)

- Flightglobal (Ascend Database)

- French Bureau d'Enquêtes et d'Analyses pour la sécurité de l'aviation civile (BEA)

- German Bundesstelle für Flugunfalluntersuchung (BFU)

- International Civil Aviation Organization (ICAO)

- Irish Air Accident Investigation Unit (AAIU)

- National Transportation Safety Board (NTSB)

Following identification from the databases, the accident / incident reports were reviewed using all available data. Where possible, the national investigative agency report was reviewed, even if that agency's database was not available for searching.

Each accident and incident is identified by ICAO (or FAA) operator code and flight number. If the flight number is not available, the last two characters of the aircraft registration will replace the flight number. If no operator code is available (i. e. non-airline flights), the full aircraft registration is used for identification.

A total of 275 accidents and incidents were identified resulting in 7185 onboard fatalities with an additional 235 ground fatalities. Forty-one percent happened at night and forty-three percent occurred during instrument meteorological conditions (IMC). Table 1a shows a decreasing trend over the period. Table $1 \mathrm{~b}$ shows the distribution by aircraft class and Table 1c shows the distribution to type of operation. Table 1d shows the distribution of events over phases of flight.

The set of accidents and incidents is provided in Appendix A.

Table 1a. Loss of Control Events Grouped by Five Year Interval

\begin{tabular}{|l|c|c|}
\hline Region & Events & $\begin{array}{l}\text { On-Board } \\
\text { Fatalities }\end{array}$ \\
\hline 1996 to 2000 & 102 & 2938 \\
\hline 2001 to 2005 & 99 & 2143 \\
\hline 2006 to 2010 & 74 & 2104 \\
\hline Total & 275 & 7185 \\
\hline
\end{tabular}

Table 1b. Loss of Control Events Grouped by Aircraft Classification

\begin{tabular}{|l|c|c|}
\hline $\begin{array}{l}\text { Aircraft } \\
\text { Classification }\end{array}$ & Events & $\begin{array}{l}\text { On-Board } \\
\text { Fatalities }\end{array}$ \\
\hline Wide-body Turbojets & 38 & 2224 \\
\hline Narrow-body Turbojets & 96 & 3858 \\
\hline Business Jets & 42 & 115 \\
\hline Turboprop Transports & 44 & 615 \\
\hline Piston Transports & 5 & 34 \\
\hline Commuter Airplanes & 50 & 339 \\
\hline Total & 275 & 7185 \\
\hline
\end{tabular}

Table 1c. Loss of Control Events Grouped by Type of Operation

\begin{tabular}{|l|c|c|}
\hline Operation & Events & $\begin{array}{l}\text { On-Board } \\
\text { Fatalities }\end{array}$ \\
\hline Scheduled Airlines & 143 & 5803 \\
\hline Non-Scheduled & 87 & 1234 \\
\hline Non-Revenue Operations & 28 & 78 \\
\hline Executive Transportation & 17 & 70 \\
\hline Total & 275 & 7185 \\
\hline
\end{tabular}

American Institute of Aeronautics and Astronautics 
Table 1d. Loss of Control Events Grouped by Phase of Flight

\begin{tabular}{|l|c|c|}
\hline Region & Events & $\begin{array}{l}\text { On-Board } \\
\text { Fatalities }\end{array}$ \\
\hline Takeoff & 6 & 270 \\
\hline Initial Climb & 79 & 1241 \\
\hline Climb & 43 & 1697 \\
\hline Cruise & 41 & 2008 \\
\hline Descent & 17 & 156 \\
\hline Holding & 22 & 0 \\
\hline Approach & 34 & 1087 \\
\hline VFR Pattern & 8 & 69 \\
\hline Circling & 3 & 152 \\
\hline Final Approach & 9 & 77 \\
\hline Landing & 18 & 37 \\
\hline Go-around & 8 & 15 \\
\hline Missed Approach & 5 & 374 \\
\hline Maneuvering & 2 & 2 \\
\hline Total & 275 & 7185 \\
\hline
\end{tabular}

\section{B. Accident / Incident Statistics by Causal and Contributing Factors}

A preliminary analysis was performed for the 275 accident / incident set defined above by dividing the set into subsets and allocating the subsets to the analysis team members. The initial step in the analysis consisted of a review of each event in the set. The level of detail in analyzing each accident and incident was therefore dependent on the level of detail provided in the accident and incident reports. Information from each report was transcribed into a categorized set of causal and contributing factors, using the categories and sub-categories defined in Fig. 1. A basic statistical summary of the accident / incident set in terms of individual LOC precursors (i.e., causal and contributing factors) is provided in Table 2. Table 2a summarizes the number of events and fatalities by precursor category and sub-category, and Tables $2 \mathrm{~b}-2 \mathrm{~d}$ provide these statistics for each individual precursor within each category and sub-category. It should be noted in Table 2 that the precursors are not mutually exclusive. For example, 240 LOC events involved one or more adverse onboard conditions, and the frequency of each sub-category within this category is listed. These numbers do not add up to 240, however, because there were many events involving more than one sub-category. Similarly, adding the number of accidents listed for the three categories exceeds the 275 total because many events involved multiple categories. The same is true for Tables $2 \mathrm{~b}-2 \mathrm{~d}$ for individual precursors.

Table 2a. Contributions to LOC Accidents and Fatalities Category \& Sub-Category Totals

\begin{tabular}{|c|c|c|c|c|}
\hline Precursor & $\begin{array}{c}\text { Accidents / } \\
\text { Incidents }\end{array}$ & $\mathbf{\%}$ & Fatalities & $\mathbf{\%}$ \\
\hline Adverse Onboard Conditions & $\mathbf{2 4 0}$ & $\mathbf{8 7 . 2 7}$ & $\mathbf{6 7 5 0}$ & $\mathbf{9 3 . 9 5}$ \\
\hline Vehicle Impairment & 86 & 31.6427 & 2576 & 35.85 \\
\hline System \& Component Failures / Malfunctions & 117 & 42.55 & 3150 & 43.84 \\
\hline Inappropriate Crew Action / Inaction & 160 & 58.18 & 4444 & 61.85 \\
\hline
\end{tabular}




\begin{tabular}{|r|c|c|c|c|}
\hline External Hazards \& Disturbances & $\mathbf{1 0 1}$ & $\mathbf{3 6 . 7 3}$ & $\mathbf{3 0 3 6}$ & $\mathbf{4 2 . 2 5}$ \\
\hline Pnclement Weather \& Atmospheric Disturbances & 65 & 23.64 & 1741 & 24.23 \\
\hline Obstacle & 16 & 10.91 & 1324 & 18.43 \\
\hline $\begin{array}{l}\text { Abnormal Dynamics \& Vehicle Upset } \\
\text { Conditions }\end{array}$ & $\mathbf{2 2 0}$ & $\mathbf{8 0 . 0 0}$ & $\mathbf{5 4 1 6}$ & $\mathbf{7 5 . 3 8}$ \\
\hline Abnormal Vehicle Dynamics & 47 & 17.09 & 312 & 4.34 \\
\hline Vehicle Upset Conditions & 188 & 68.36 & 5315 & 73.97 \\
\hline
\end{tabular}

Table 2b. Contributions to LOC Accidents and Fatalities Adverse Onboard Conditions

\begin{tabular}{|c|c|c|c|c|}
\hline Precursor & $\begin{array}{l}\text { Accidents / } \\
\text { Incidents }\end{array}$ & $\%$ & Fatalities & $\%$ \\
\hline Adverse Onboard Conditions & 240 & 87.27 & 6750 & 93.95 \\
\hline Vehicle Impairment & 86 & 31.27 & 2576 & 35.85 \\
\hline Inappropriate Vehicle Configuration & 22 & 8.00 & 468 & 6.51 \\
\hline Contaminated Airfoil & 22 & 8.00 & 230 & 3.20 \\
\hline Improper Loading: Weight / Balance / CG Issues & 12 & 4.36 & 95 & 1.32 \\
\hline Improper Loading: Cargo Problems / Hazards & 1 & 0.36 & 110 & 1.53 \\
\hline Airframe Structural Damage & 33 & 12.00 & 1882 & 26.19 \\
\hline $\begin{array}{ll}\text { Engine Damage (FOD) } \\
\end{array}$ & 1 & 0.36 & 2 & 0.03 \\
\hline System \& Component Failures / Malfunctions & 117 & 42.55 & 3150 & 43.84 \\
\hline $\begin{array}{r}\text { System Operational Error (Design Flaw / } \\
\text { Validation Error) }\end{array}$ & 15 & 5.45 & 497 & 6.92 \\
\hline $\begin{array}{r}\text { System Operational Error (Software / Verification } \\
\text { Error) }\end{array}$ & 4 & 1.45 & 9 & 0.13 \\
\hline Control Component Failure / Malfunction & 41 & 14.91 & 426 & 5.93 \\
\hline Engine Failure / Malfunction & 37 & 13.45 & 989 & 13.76 \\
\hline Sensor / Sensor System Failure / Malfunction & 16 & 5.82 & 671 & 9.34 \\
\hline Flight Deck Instrumentation Failure / Malfunction & 11 & 4.00 & 323 & 4.50 \\
\hline $\begin{array}{r}\text { System / Subsystem Failure / Malfunction (Non- } \\
\text { control component) }\end{array}$ & 12 & 4.36 & 641 & 8.92 \\
\hline Inappropriate Crew Action / Inaction & 160 & 58.18 & 4444 & 61.85 \\
\hline $\begin{array}{r}\text { Loss of Attitude State Awareness / Spatial } \\
\text { Disorientation }\end{array}$ & 28 & 10.18 & 1513 & 21.06 \\
\hline $\begin{array}{r}\text { Loss of Energy State Awareness / Inadequate } \\
\text { Energy Management }\end{array}$ & 51 & 18.55 & 1402 & 19.51 \\
\hline $\begin{array}{r}\text { Lack of Aircraft / System State Awareness / Mode } \\
\text { Confusion }\end{array}$ & 5 & 1.82 & 11 & 0.15 \\
\hline $\begin{array}{l}\text { Aggressive Maneuver } \\
\end{array}$ & 14 & 5.09 & 313 & 4.36 \\
\hline Abnormal / Inadvertent Control Input / Maneuver & 12 & 4.36 & 605 & 8.42 \\
\hline Improper / Ineffective Recovery & 26 & 9.45 & 1164 & 16.20 \\
\hline
\end{tabular}




\begin{tabular}{|r|c|c|c|c|}
\hline $\begin{array}{r}\text { Inadequate Crew Resource Monitoring / } \\
\text { Management (PF, PNF, \& Systems) }\end{array}$ & 36 & 13.09 & 1508 & 20.99 \\
\hline Improper Procedure & 53 & 19.27 & 750 & 10.44 \\
\hline Fatigue / Impairment (Includes Hypoxia) & 9 & 3.27 & 376 & 5.23 \\
\hline
\end{tabular}

Table 2c. Contributions to LOC Accidents and Fatalities -

External Hazards \& Disturbances

\begin{tabular}{|c|c|c|c|c|}
\hline Precursor & $\begin{array}{l}\text { Accidents / } \\
\text { Incidents }\end{array}$ & $\%$ & Fatalities & $\%$ \\
\hline External Hazards \& Disturbances & 101 & 36.73 & 3036 & 42.25 \\
\hline $\begin{array}{c}\text { Inclement Weather \& Atmospheric } \\
\text { Disturbances }\end{array}$ & 65 & 23.64 & 1741 & 24.23 \\
\hline Thunderstorms / Rain & 9 & 3.27 & 611 & 8.50 \\
\hline Wind Shear & 9 & 3.27 & 249 & 3.47 \\
\hline Turbulence & 10 & 3.64 & 486 & 6.76 \\
\hline Wake Vortex & 7 & 2.55 & 284 & 3.95 \\
\hline Snow / Icing & 33 & 12.00 & 426 & 5.93 \\
\hline Poor Visibility & 30 & 10.91 & 1324 & 18.43 \\
\hline Fog / Haze & 16 & 5.82 & 502 & 6.99 \\
\hline Night & 18 & 6.55 & 915 & 12.73 \\
\hline Obstacle & 16 & 5.82 & 601 & 8.36 \\
\hline Fixed & 4 & 1.45 & 8 & 0.11 \\
\hline Moving & 12 & 4.36 & 593 & 8.25 \\
\hline
\end{tabular}

Table 2d. Contributions to LOC Accidents and Fatalities -

Abnormal Dynamics \& Vehicle Upset Conditions

\begin{tabular}{|r|c|c|c|c|}
\hline \multicolumn{1}{|c|}{ Precursor } & $\begin{array}{c}\text { Accidents / } \\
\text { Incidents }\end{array}$ & $\mathbf{\%}$ & Fatalities & $\mathbf{\%}$ \\
\hline $\begin{array}{l}\text { Abnormal Dynamics \& Vehicle Upset } \\
\text { Conditions }\end{array}$ & $\mathbf{2 2 0}$ & $\mathbf{8 0 . 0 0}$ & $\mathbf{5 4 1 6}$ & $\mathbf{7 5 . 3 8}$ \\
\hline Abnormal Vehicle Dynamics & $\mathbf{4 7}$ & $\mathbf{1 7 . 0 9}$ & $\mathbf{3 1 2}$ & $\mathbf{4 . 3 4}$ \\
\hline Uncommanded Motions & 24 & 8.73 & 89 & 1.24 \\
\hline Oscillatory Vehicle Response (Includes PIO) & 14 & 5.09 & 9 & 0.13 \\
\hline Abnormal Control for Trim / Flight & 8 & 2.91 & 14 & 0.19 \\
\hline Abnormal / Counterintuitive Control Responses & 4 & 1.45 & 200 & 2.78 \\
\hline Vehicle Upset Conditions & $\mathbf{1 8 8}$ & $\mathbf{6 8 . 3 6}$ & $\mathbf{5 3 1 5}$ & $\mathbf{7 3 . 9 7}$ \\
\hline Abnormal Attitude & 29 & 10.55 & 856 & 11.91 \\
\hline Abnormal Airspeed (Includes Low Energy) & 24 & 8.73 & 750 & 10.44 \\
\hline Abnormal Angular Rates & 1 & 0.36 & 10 & 0.14 \\
\hline Undesired Abrupt Dynamic Response & 18 & 6.55 & 218 & 3.03 \\
\hline Abnormal Flight Trajectory & 26 & 9.45 & 1055 & 14.68 \\
\hline Uncontrolled Descent (Includes Spiral Dive) & 47 & 17.09 & 1534 & 21.35 \\
\hline Stall / Departure (Includes Falling Leaf, Spin) & 74 & 26.91 & 1796 & 25.00 \\
\hline
\end{tabular}

8

American Institute of Aeronautics and Astronautics 
Table 2 is useful for determining the number of events and fatalities associated with individual causal and contributing factors, but it does not provide any information on combinations or sequencing of these factors. Nonetheless, this table identifies System \& Component Failures and Malfunctions, Inappropriate Crew Action and Inaction, and Vehicle Upsets as the largest sub-category contributors to the number of events and fatalities within the accident / incident set evaluated. Other key contributors included Vehicle Impairment, Inclement Weather and Atmospheric Disturbances, and Abnormal Vehicle Dynamics. The following subsections C and D address combinations and sequencing of LOC causal and contributing factors, respectively.

\section{Worst-Case Precursor Combinations}

A preliminary analysis of the accident / incident set in terms of worst-case combinations of causal and contributing factors (as defined by number of accidents and resulting fatalities), was determined using threedimensional scatter plots. The three dimensions are aligned with the three categories identified in Table 2. Sphere size is directly proportional to the number of accidents, and sphere color depicts the number of fatalities as indicated by the legend. Figure 3 shows scatter plots by category and sub-category with and without within-category overlap. Fig. 3a shows worst-case precursor sub-category combinations and includes within-category overlap. For example, combinations involving system failures / malfunctions do not exclude cases that also involved inappropriate crew actions / inactions. Fig. 3b excludes within-category overlap. The team felt that excluding cases of multiple withincategory precursors resulted in unacceptable loss of information, so it was determined that the analysis should include this overlap. All remaining figures in this paper therefore include within-category overlap. As indicated by Fig. 3a, precursor combinations involving system failure / malfunction, inappropriate crew action / inaction, and vehicle upset conditions led to the highest number of fatalities both with and without involvement by inclement weather / atmospheric disturbance and poor visibility. Vehicle impairment with and without vehicle upsets also led to a high level of fatalities. These worst-case sub-category combinations can be further explored by generating scatter plots within these sub-categories. For example, Figure 4 shows a precursor level scatter plot to investigate the specific precursors that contributed to the "Inappropriate Crew Action / Inaction" - "Poor Visibility" - "Vehicle Upset" combination of Figure 3a. As indicated in Figure 4b, the precursors that contributed to this sub-category combination were entirely "Loss of Attitude State Awareness" and "Loss of Energy State Awareness", predominantly at night, and leading primarily to abnormal trajectories, uncontrolled descent, and stall/departure. Additional worst-case precursor-level evaluations will be performed for the final analysis.

Figures 5a and 5b present scatter plots that separate non-fly-by-wire (non-FBW) and fly-by-wire (FBW) aircraft, respectively. Although there were only 24 accidents / incidents in the data set involving FBW aircraft, it is interesting to investigate as a separate group. The results for non-FBW aircraft are very similar to the full set. The FBW aircraft analysis identifies system failure / malfunction and vehicle impairment combined with vehicle upset as the worst-case sub-category combinations. 


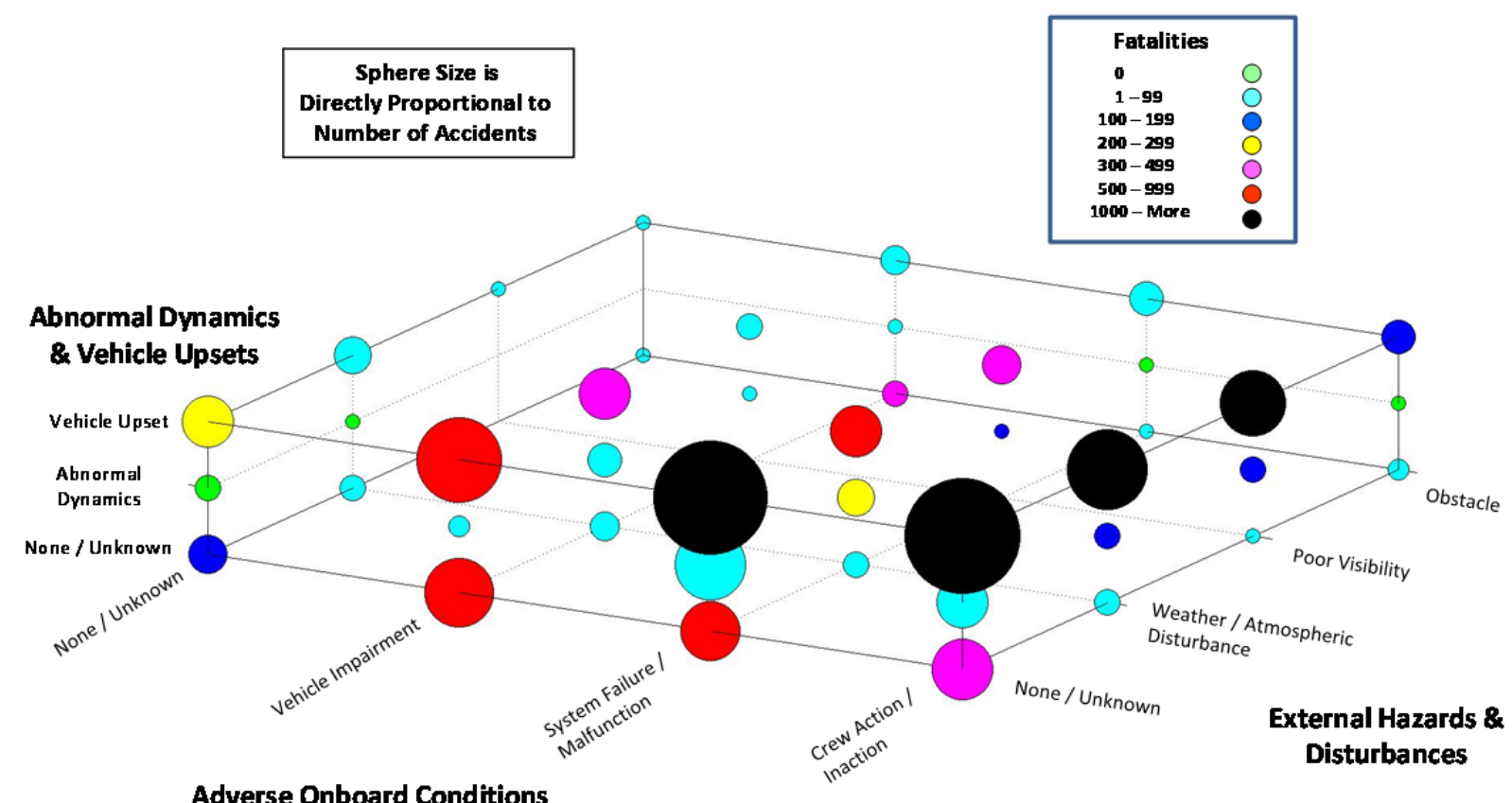

Figure 3a. Worst-Case Combinations of LOC Precursor Sub-Categories, with Within-Category Overlap.

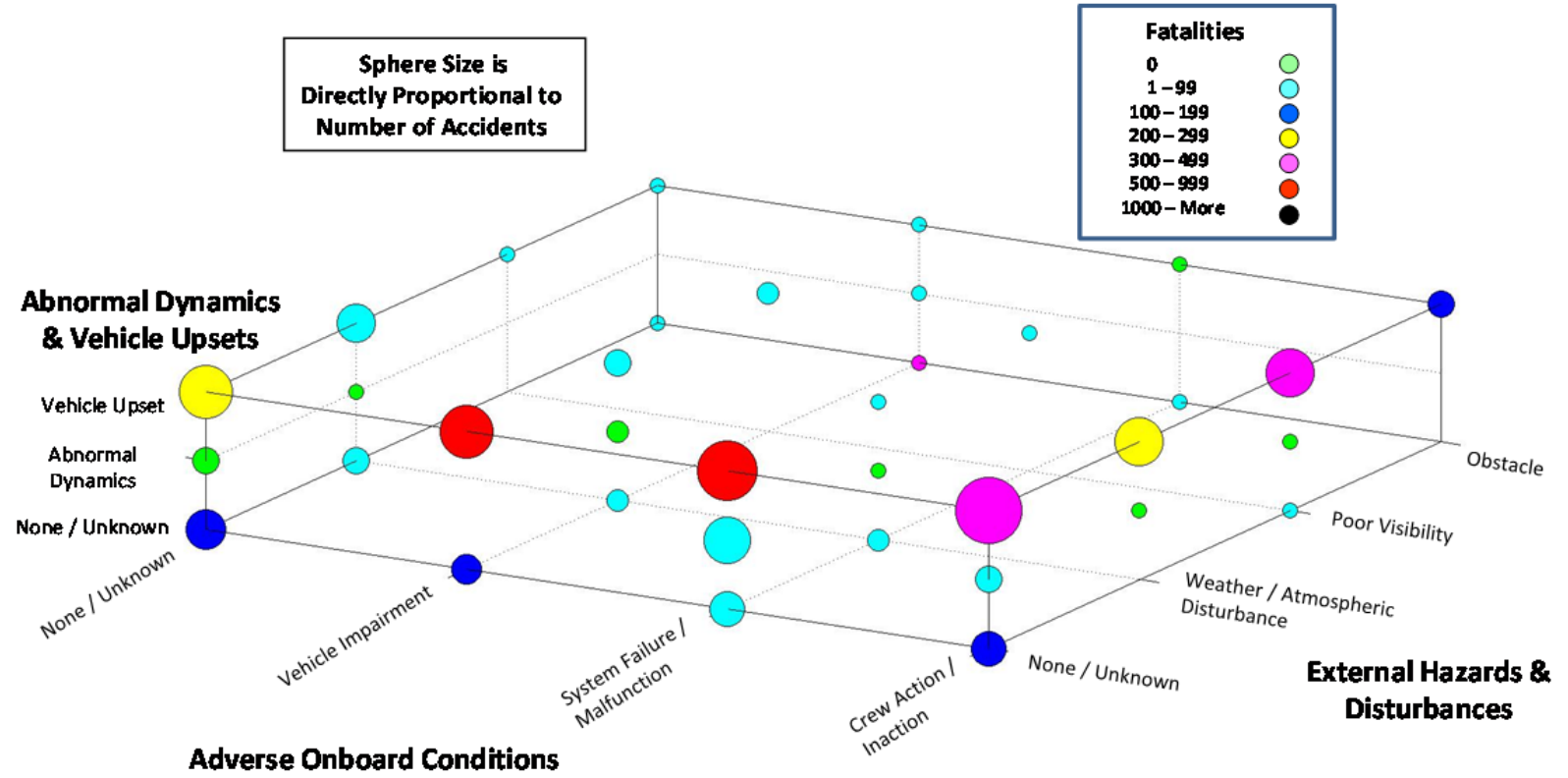

Figure 3b. Worst-Case Combinations of LOC Precursor Sub-Categories, without Within-Category Overlap. 


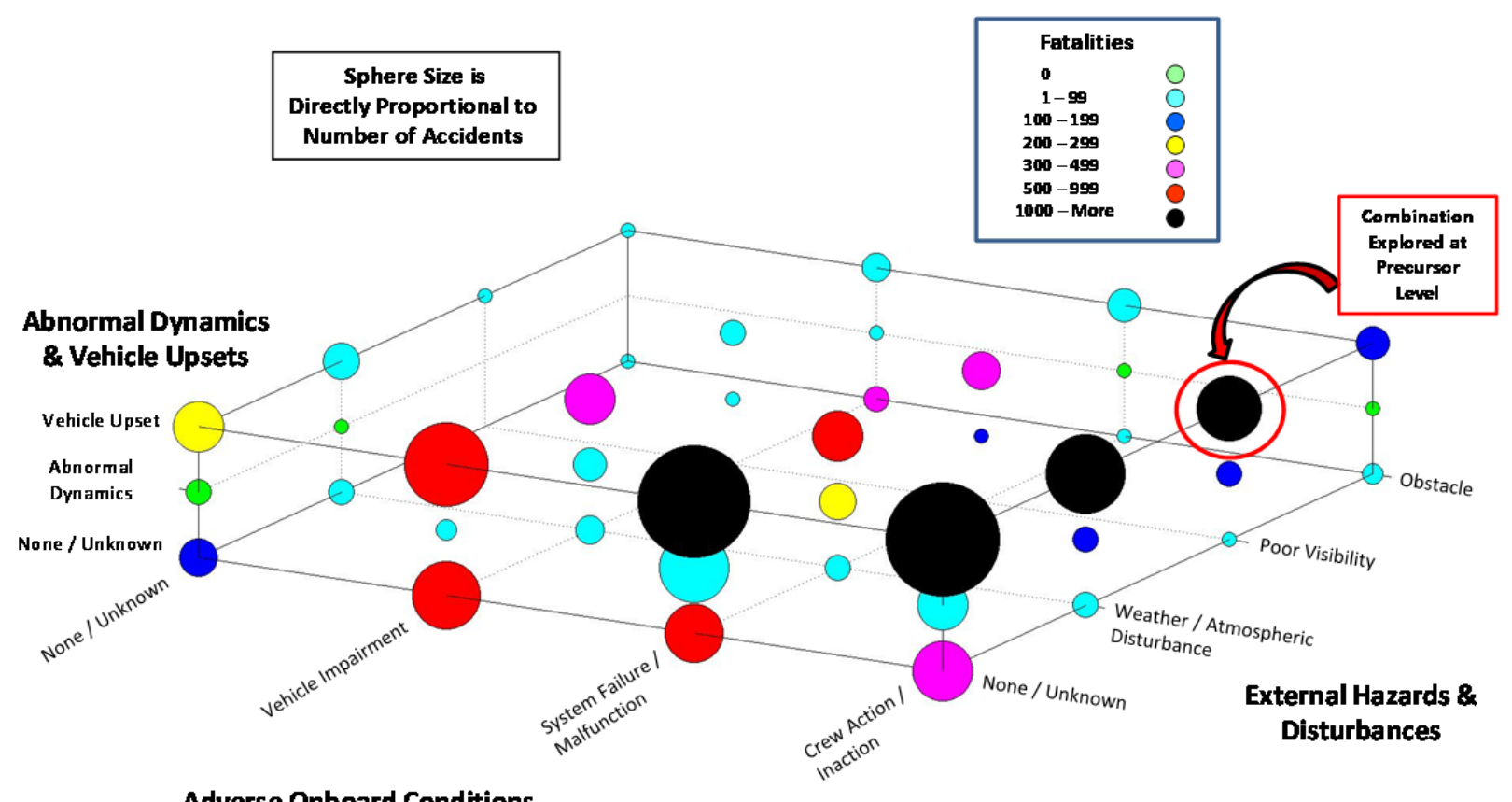

Adverse Onboard Conditions

Figure 4a. Example Sub-Category Combination Explored at Precursor Level in Figure 4b.

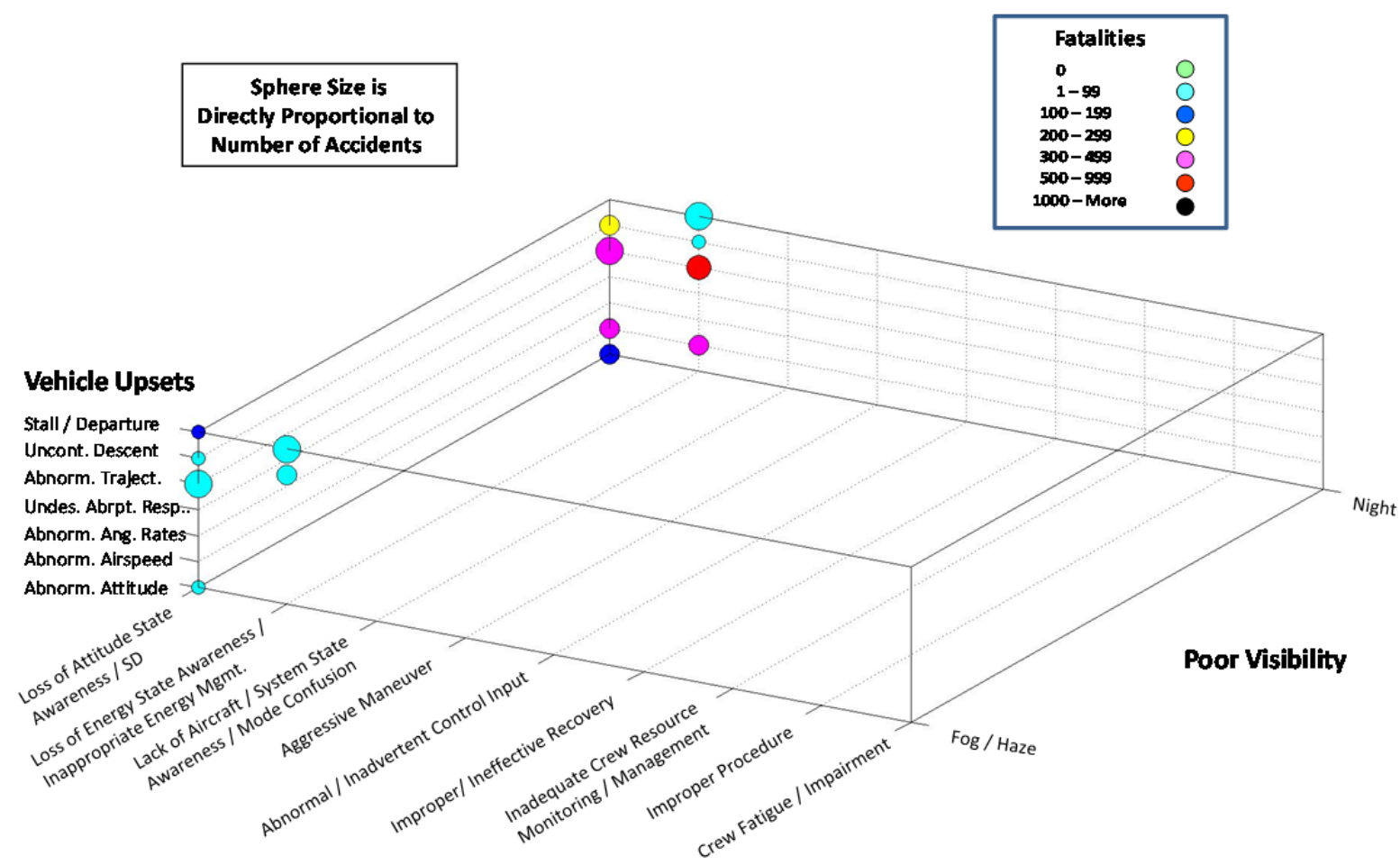

Inappropriate Crew Action / Inaction

Figure 4b. Precursor Combinations within Sub-Category Combination of Figure 4a. 


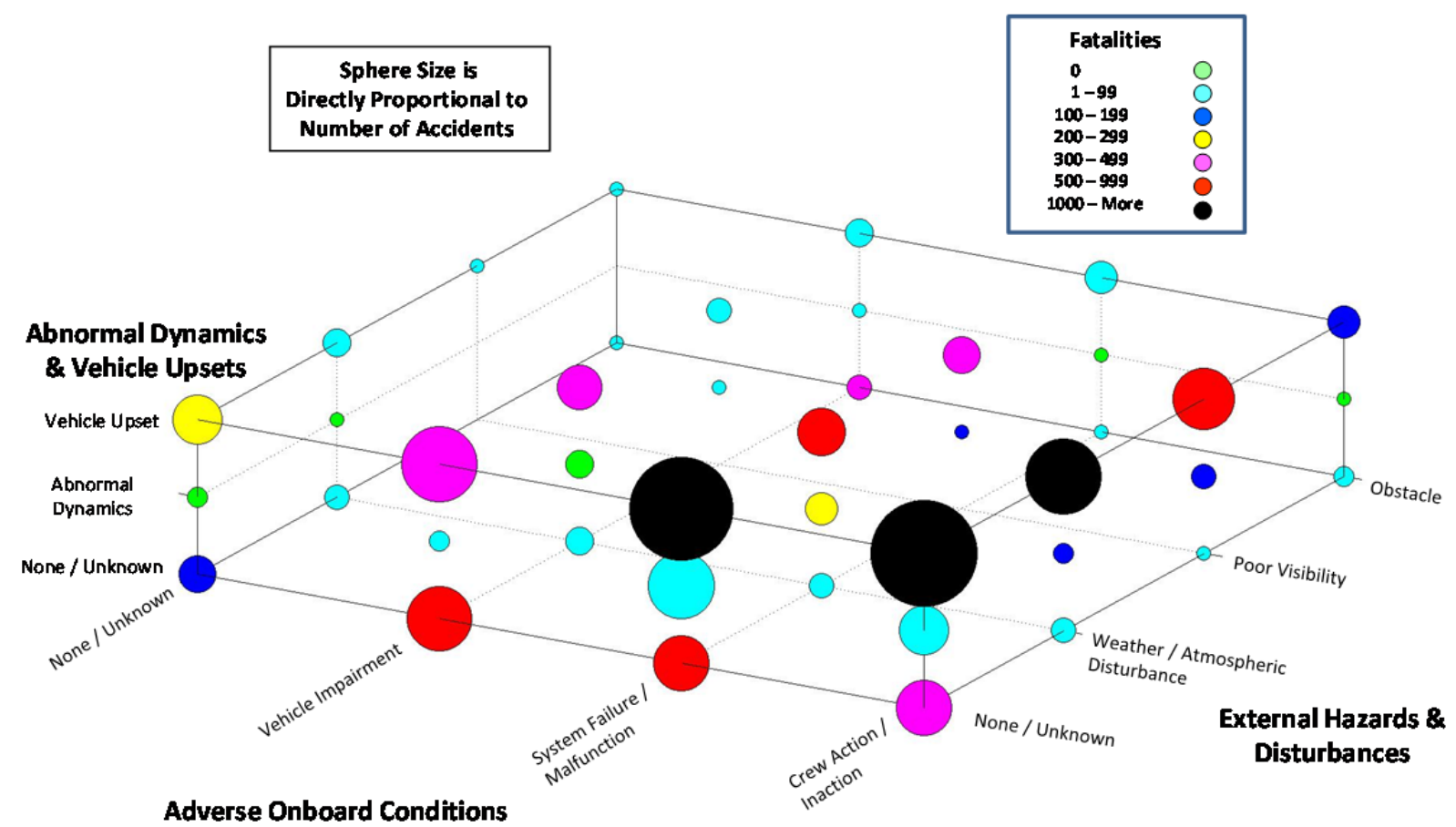

Figure 5a. Worst-Case Combinations of LOC Precursor Sub-Categories for Non-FBW Aircraft.

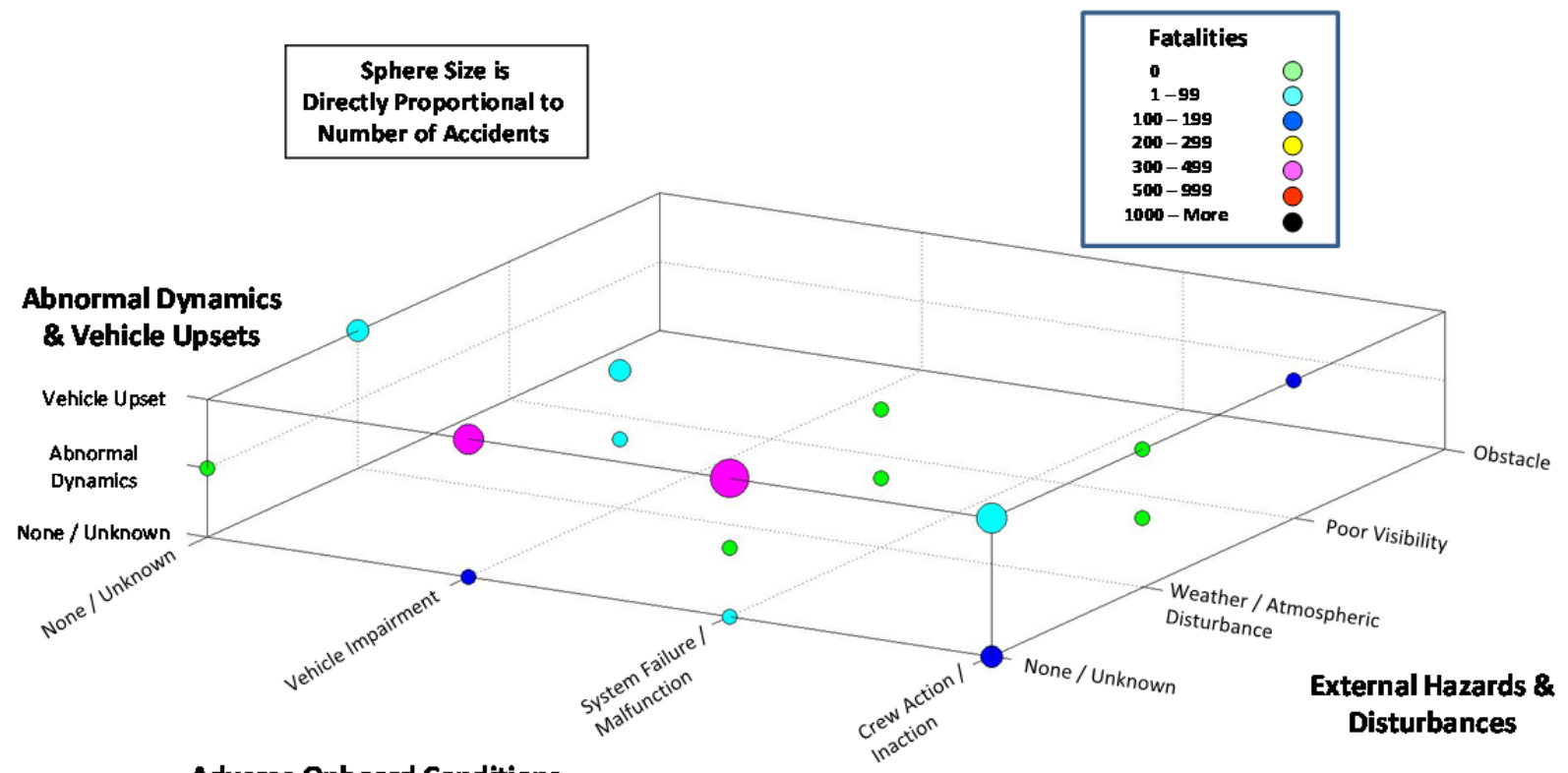

Adverse Onboard Conditions

Figure 5b. Worst-Case Combinations of LOC Precursor Sub-Categories for FBW Aircraft. 


\section{Precursor Sequences}

A preliminary analysis of precursor temporal ordering was completed to identify dominant precursors and worst case sequences for the accidents and incidents of Appendix A. Table 3a shows a summary of the sequential occurrences for each precursor category and sub-category, and Tables $3 b-3 d$ show sequence summaries for each precursor in each sub-category. As shown, up to seven precursors were identified on several events but most of the accidents and incidents had no more than five.

Table 3a. Sequencing of LOC Accident Precursors Category \& Sub-Category Totals

\begin{tabular}{|c|c|c|c|c|c|c|c|}
\hline Precursor & $\mathbf{1 s t}$ & 2nd & 3rd & 4th & 5th & 6th & 7th \\
\hline Adverse Onboard Conditions & $\mathbf{1 6 7}$ & $\mathbf{1 5 3}$ & $\mathbf{8 8}$ & $\mathbf{3 9}$ & $\mathbf{1 0}$ & $\mathbf{3}$ & $\mathbf{0}$ \\
\hline Vehicle Impairment & 41 & 32 & 11 & 4 & 3 & 0 & 0 \\
\hline System \& Component Failures / Malfunctions & 84 & 35 & 10 & 5 & 1 & 0 & 0 \\
\hline Inappropriate Crew Action / Inaction & 42 & 86 & 67 & 30 & 6 & 3 & 0 \\
\hline External Hazards \& Disturbances & $\mathbf{8 6}$ & $\mathbf{1 6}$ & $\mathbf{4}$ & $\mathbf{2}$ & $\mathbf{0}$ & $\mathbf{1}$ & $\mathbf{0}$ \\
\hline Inclement Weather \& Atmospheric Disturbances & 58 & 6 & 1 & 1 & 0 & 0 & 0 \\
\hline Poor Visibility & 19 & 6 & 2 & 0 & 0 & 0 & 0 \\
\hline Obstacle & 9 & 4 & 1 & 1 & 0 & 1 & 0 \\
\hline $\begin{array}{r}\text { Abnormal Dynamics \& Vehicle Upset } \\
\text { Conditions }\end{array}$ & $\mathbf{0}$ & $\mathbf{8 9}$ & $\mathbf{7 8}$ & $\mathbf{5 5}$ & $\mathbf{3 3}$ & $\mathbf{1 1}$ & $\mathbf{3}$ \\
\hline Abnormal Vehicle Dynamics & 0 & 23 & 14 & 8 & 4 & 1 & 0 \\
\hline Vehicle Upset Conditions & 0 & 66 & 64 & 47 & 29 & 10 & 3 \\
\hline Unknown Precipitating Events & $\mathbf{2 2}$ & - & - & - & - & - & - \\
\hline
\end{tabular}

Table 3b. Sequencing of LOC Accident Precursors Adverse Onboard Conditions

\begin{tabular}{|r|c|c|c|c|c|c|c|}
\hline Precursor & $\mathbf{1 s t}$ & $\mathbf{2 n d}$ & $\mathbf{3 r d}$ & $\mathbf{4 t h}$ & $\mathbf{5 t h}$ & $\mathbf{6 t h}$ & $\mathbf{7 t h}$ \\
\hline Adverse Onboard Conditions & $\mathbf{1 6 7}$ & $\mathbf{1 5 3}$ & $\mathbf{8 8}$ & $\mathbf{3 9}$ & $\mathbf{1 0}$ & $\mathbf{3}$ & $\mathbf{0}$ \\
\hline Vehicle Impairment & $\mathbf{4 1}$ & $\mathbf{3 2}$ & $\mathbf{1 1}$ & $\mathbf{4}$ & $\mathbf{3}$ & $\mathbf{0}$ & $\mathbf{0}$ \\
\hline Inappropriate Vehicle Configuration & 13 & 6 & 2 & 1 & 0 & 0 & 0 \\
\hline Contaminated Airfoil & 2 & 18 & 1 & 0 & 1 & 0 & 0 \\
\hline Improper Loading: Weight / Balance / CG Issues & 10 & 2 & 0 & 0 & 0 & 0 & 0 \\
\hline Improper Loading: Cargo Problems / Hazards & 1 & 0 & 0 & 0 & 0 & 0 & 0 \\
\hline Airframe Structural Damage & 14 & 6 & 8 & 3 & 2 & 0 & 0 \\
\hline Engine Damage (FOD) & 1 & 0 & 0 & 0 & 0 & 0 & 0 \\
\hline System \& Component Failures / Malfunctions & $\mathbf{8 4}$ & $\mathbf{3 5}$ & $\mathbf{1 0}$ & $\mathbf{5}$ & $\mathbf{1}$ & $\mathbf{0}$ & $\mathbf{0}$ \\
\hline System Operational Error (Design Flaw / Validation Error) & 12 & 1 & 2 & 0 & 0 & 0 & 0 \\
\hline System Operational Error (Software / Verification Error) & 2 & 1 & 1 & 0 & 0 & 0 & 0 \\
\hline
\end{tabular}




\begin{tabular}{|c|c|c|c|c|c|c|c|}
\hline Control Component Failure / Malfunction & 26 & 10 & 3 & 2 & 0 & 0 & 0 \\
\hline Engine Failure / Malfunction & 27 & 7 & 0 & 2 & 1 & 0 & 0 \\
\hline Sensor / Sensor System Failure / Malfunction & 6 & 10 & 0 & 0 & 0 & 0 & 0 \\
\hline Flight Deck Instrumentation Failure / Malfunction & 4 & 3 & 2 & 1 & 0 & 0 & 0 \\
\hline $\begin{array}{r}\text { System / Subsystem Failure / Malfunction ( } \begin{array}{r}\text { Non-control } \\
\text { component) }\end{array} \\
\end{array}$ & 7 & 3 & 2 & 0 & 0 & 0 & 0 \\
\hline Inappropriate Crew Action / Inaction & 42 & 86 & 67 & 30 & 6 & 3 & $\mathbf{0}$ \\
\hline Loss of Attitude State Awareness / Spatial Disorientation & 3 & 8 & 11 & 3 & 1 & 2 & 0 \\
\hline $\begin{array}{r}\text { Loss of Energy State Awareness / Inadequate Energy } \\
\text { Management }\end{array}$ & 9 & 16 & 18 & 8 & 0 & 0 & 0 \\
\hline $\begin{array}{r}\text { Lack of Aircraft / System State Awareness / Mode } \\
\text { Confusion }\end{array}$ & 1 & 3 & 1 & 0 & 0 & 0 & 0 \\
\hline $\begin{array}{l}\text { Aggressive Maneuver } \\
\end{array}$ & 2 & 7 & 2 & 3 & 0 & 0 & 0 \\
\hline Abnormal / Inadvertent Control Input / Maneuver & 4 & 3 & 3 & 2 & 0 & 0 & 0 \\
\hline Improper / Ineffective Recovery & 0 & 7 & 10 & 5 & 3 & 1 & 0 \\
\hline $\begin{array}{r}\text { Inadequate Crew Resource Monitoring / Management (PF, } \\
\text { PNF, \& Systems) }\end{array}$ & 4 & 16 & 11 & 3 & 2 & 0 & 0 \\
\hline Improper Procedure & 17 & 22 & 10 & 4 & 0 & 0 & 0 \\
\hline Fatigue / Impairment (Includes Hypoxia) & 2 & 4 & 1 & 2 & 0 & 0 & 0 \\
\hline
\end{tabular}

Table 3c. Sequencing of LOC Accident Precursors External Hazards \& Disturbances

\begin{tabular}{|c|c|c|c|c|c|c|c|}
\hline Precursor & 1st & 2nd & 3rd & 4th & 5th & 6th & 7th \\
\hline External Hazards \& Disturbances & 86 & 16 & 4 & 2 & $\mathbf{0}$ & 1 & $\mathbf{0}$ \\
\hline $\begin{array}{l}\text { Inclement Weather \& Atmospheric } \\
\text { Disturbances }\end{array}$ & 58 & 6 & 1 & 1 & $\mathbf{0}$ & $\mathbf{0}$ & $\mathbf{0}$ \\
\hline Thunderstorms / Rain & 8 & 1 & 0 & 0 & 0 & 0 & 0 \\
\hline Wind Shear & 6 & 1 & 1 & 0 & 0 & 0 & 0 \\
\hline Turbulence & 5 & 4 & 0 & 0 & 0 & 0 & 0 \\
\hline Wake Vortex & 7 & 0 & 0 & 0 & 0 & 0 & 0 \\
\hline Snow / Icing & 32 & 0 & 0 & 1 & 0 & 0 & 0 \\
\hline Poor Visibility & 19 & 6 & 2 & $\mathbf{0}$ & $\mathbf{0}$ & $\mathbf{0}$ & $\mathbf{0}$ \\
\hline Fog / Haze & 8 & 4 & 1 & 0 & 0 & 0 & 0 \\
\hline Night & 11 & 2 & 1 & 0 & 0 & 0 & 0 \\
\hline Obstacle & 9 & 4 & 1 & 1 & $\mathbf{0}$ & 1 & $\mathbf{0}$ \\
\hline Fixed & 2 & 0 & 0 & 1 & 0 & 1 & 0 \\
\hline Moving & 7 & 4 & 1 & 0 & 0 & 0 & 0 \\
\hline
\end{tabular}


Table 3d. Sequencing of LOC Accidents Abnormal Dynamics \& Vehicle Upset Conditions

\begin{tabular}{|r|c|c|c|c|c|c|c|}
\hline \multicolumn{1}{|c|}{ Precursor } & $\mathbf{1 s t}$ & $\mathbf{2 n d}$ & $\mathbf{3 r d}$ & $\mathbf{4 t h}$ & $\mathbf{5 t h}$ & $\mathbf{6 t h}$ & $\mathbf{7 t h}$ \\
\hline $\begin{array}{r}\text { Abnormal Dynamics \& Vehicle Upset } \\
\text { Conditions }\end{array}$ & $\mathbf{0}$ & $\mathbf{8 9}$ & $\mathbf{7 8}$ & $\mathbf{5 5}$ & $\mathbf{3 3}$ & $\mathbf{1 1}$ & $\mathbf{3}$ \\
\hline Abnormal Vehicle Dynamics & $\mathbf{0}$ & $\mathbf{2 3}$ & $\mathbf{1 4}$ & $\mathbf{8}$ & $\mathbf{4}$ & $\mathbf{1}$ & $\mathbf{0}$ \\
\hline Uncommanded Motions & 0 & 15 & 6 & 3 & 0 & 0 & 0 \\
\hline Oscillatory Vehicle Response (Includes PIO) & 0 & 4 & 2 & 4 & 3 & 1 & 0 \\
\hline Abnormal Control for Trim / Flight & 0 & 2 & 5 & 0 & 1 & 0 & 0 \\
\hline Abnormal / Counterintuitive Control Responses & 0 & 2 & 1 & 1 & 0 & 0 & 0 \\
\hline Vehicle Upset Conditions & $\mathbf{0}$ & $\mathbf{6 6}$ & $\mathbf{6 4}$ & $\mathbf{4 7}$ & $\mathbf{2 9}$ & $\mathbf{1 0}$ & $\mathbf{3}$ \\
\hline Abnormal Attitude & 0 & 8 & 12 & 5 & 3 & 0 & 1 \\
\hline Abnormal Airspeed (Includes Low Energy) & 0 & 8 & 6 & 6 & 2 & 2 & 0 \\
\hline Abnormal Angular Rates & 0 & 1 & 0 & 0 & 0 & 0 & 0 \\
\hline Abnormal Flight Trajectory & 0 & 9 & 3 & 5 & 6 & 1 & 2 \\
\hline Undesired Abrupt Dynamic Response & 0 & 8 & 6 & 2 & 1 & 1 & 0 \\
\hline Stall / Departure (Includes Falling Leaf, Spin) & 0 & 14 & 26 & 17 & 14 & 3 & 0 \\
\hline
\end{tabular}

Tables 3a-d indicate that LOC events were usually first precipitated by an adverse onboard condition and most often by a system or component failure or malfunction. The second precipitating factor occurring most often was an external hazard or disturbance and in that case usually related to weather or reduced visibility. Moreover, external hazards and disturbances rarely occurred further downstream than $2^{\text {nd }}$ in LOC sequences. Vehicle upsets were rarely the initial factor but rather an outcome of an external hazard/disturbance or adverse onboard condition.

Within "Adverse Onboard Conditions", inappropriate crew action / inaction and vehicle impairment were equally likely to be a precipitating factor but inappropriate crew action / inaction occurred as the second or third event in response to a precipitating factor. Adverse onboard conditions were also the most likely factor to occur second in temporal sequencing leading to aircraft LOC. Within this category inappropriate crew action / inaction was the most likely secondary factor to occur indicating crew response to some precipitating event. Vehicle impairment or system and component failure were equally likely to be the second factor where contaminated airfoil was the leading cause of impairment.

Within "External Hazards and Disturbances", the leading initial factor was snow / icing, followed by nearly equal occurrences of wind shear, turbulence, wake vortex, and thunderstorms. It is also noteworthy that external hazards or disturbances were most often a precipitating event. Vehicle upsets most often occurred as the second, third, or fourth factor in the LOC sequence indicating that multiple precursors can lead to an upset.

Tables 4a-c summarize the number of accidents and fatalities associated with each initiating precursor. 
Table 4a. Summary of LOC Accidents \& Fatalities by Initiating Factors Factor Category \& Sub-Category Totals

\begin{tabular}{|c|c|c|c|c|}
\hline First Precursor in LOC Sequence & $\begin{array}{c}\text { Accidents / } \\
\text { Incidents } \\
\end{array}$ & $\%$ & Fatalities & $\%$ \\
\hline Adverse Onboard Conditions & 167 & 60.73 & 4099 & 57.05 \\
\hline Vehicle Impairment & 41 & 14.91 & 1153 & 16.05 \\
\hline System \& Component Failures / Malfunctions & 84 & 30.55 & 2175 & 30.27 \\
\hline Inappropriate Crew Action / Inaction & 42 & 15.27 & 771 & 10.73 \\
\hline External Hazards \& Disturbances & 86 & 31.27 & 2708 & 37.69 \\
\hline Inclement Weather \& Atmospheric Disturbances & 58 & 21.09 & 1390 & 19.35 \\
\hline Poor Visibility & 19 & 6.91 & 832 & 11.58 \\
\hline Obstacle & 9 & 3.27 & 486 & 6.76 \\
\hline $\begin{array}{l}\text { Abnormal Dynamics \& Vehicle Upset } \\
\text { Conditions }\end{array}$ & $\mathbf{0}$ & $\mathbf{0}$ & $\mathbf{0}$ & $\mathbf{0}$ \\
\hline Abnormal Vehicle Dynamics & 0 & 0 & 0 & 0 \\
\hline Vehicle Upset Conditions & 0 & 0 & 0 & 0 \\
\hline Unknown & 22 & 8.00 & 378 & 5.26 \\
\hline TOTAL & 275 & 100 & 7185 & 100 \\
\hline
\end{tabular}

Table 4b. Summary of LOC Accidents \& Fatalities by Initiating Factors Adverse Onboard Conditions

\begin{tabular}{|c|c|c|c|c|}
\hline First Precursor in LOC Sequence & $\begin{array}{c}\text { Accidents / } \\
\text { Incidents }\end{array}$ & $\%$ & Fatalities & $\%$ \\
\hline Adverse Onboard Conditions & 167 & 60.73 & 4099 & 57.05 \\
\hline Vehicle Impairment & 41 & 14.91 & 1153 & 16.05 \\
\hline Inappropriate Vehicle Configuration & 13 & 4.73 & 206 & 2.87 \\
\hline Contaminated Airfoil & 2 & 0.73 & 55 & 0.76 \\
\hline Improper Loading: Weight / Balance / CG Issues & 10 & 3.64 & 69 & 0.96 \\
\hline Improper Loading: Cargo Problems / Hazards & 1 & 0.36 & 110 & 1.53 \\
\hline Airframe Structural Damage & 14 & 5.09 & 711 & 9.89 \\
\hline $\begin{array}{l}\text { Engine Damage (FOD) } \\
\end{array}$ & 1 & 0.36 & 2 & 0.03 \\
\hline System \& Component Failures / Malfunctions & 84 & 30.55 & 2175 & 30.27 \\
\hline $\begin{array}{r}\text { System Operational Error (Design Flaw / } \\
\text { Validation Error) } \\
\end{array}$ & 12 & 4.36 & 497 & 6.92 \\
\hline $\begin{array}{r}\text { System Operational Error (Software / Verification } \\
\text { Error) }\end{array}$ & 2 & 0.73 & 0 & 0 \\
\hline Control Component Failure / Malfunction & 26 & 9.45 & 257 & 3.58 \\
\hline $\begin{array}{r}\text { Engine Failure / Malfunction } \\
\end{array}$ & 27 & 9.82 & 548 & 7.63 \\
\hline Sensor / Sensor System Failure / Malfunction & 6 & 2.18 & 198 & 2.76 \\
\hline Flight Deck Instrumentation Failure / Malfunction & 4 & 1.45 & 298 & 4.15 \\
\hline $\begin{array}{r}\text { System / Subsystem Failure / Malfunction (Non- } \\
\text { control component) }\end{array}$ & 7 & 2.55 & 377 & 5.25 \\
\hline
\end{tabular}




\begin{tabular}{|r|c|c|c|c|}
\hline Inappropriate Crew Action / Inaction & $\mathbf{4 2}$ & $\mathbf{1 5 . 2 7}$ & $\mathbf{7 7 1}$ & $\mathbf{1 0 . 7 3}$ \\
\hline $\begin{array}{r}\text { Loss of Attitude State Awareness / Spatial } \\
\text { Disorientation }\end{array}$ & 3 & 1.09 & 158 & 2.20 \\
\hline $\begin{array}{r}\text { Loss of Energy State Awareness / Inadequate } \\
\text { Energy Management }\end{array}$ & 9 & 3.27 & 152 & 2.12 \\
\hline $\begin{array}{r}\text { Lack of Aircraft / System State Awareness / Mode } \\
\text { Confusion }\end{array}$ & 1 & 0.36 & 0 & 0 \\
\hline Aggressive Maneuver & 2 & 0.73 & 4 & 0.06 \\
\hline Abnormal / Inadvertent Control Input / Maneuver & 4 & 1.45 & 115 & 1.60 \\
\hline Improper / Ineffective Recovery & 0 & 0 & 0 & 0 \\
\hline $\begin{array}{r}\text { Inadequate Crew Resource Monitoring / } \\
\text { Management (PF, PNF, \& Systems) }\end{array}$ & 4 & 1.45 & 22 & 0.31 \\
\hline Improper Procedure & 17 & 6.18 & 302 & 4.20 \\
\hline Fatigue / Impairment (Includes Hypoxia) & 2 & 0.73 & 18 & 0.25 \\
\hline
\end{tabular}

Table 4c. Summary of LOC Accidents \& Fatalities by Initiating Factors External Hazards \& Disturbances

\begin{tabular}{|c|c|c|c|c|}
\hline First Precursor in LOC Sequence & $\begin{array}{c}\text { Accidents / } \\
\text { Incidents }\end{array}$ & $\%$ & Fatalities & $\%$ \\
\hline External Hazards \& Disturbances & 86 & 31.27 & 2708 & 37.69 \\
\hline $\begin{array}{c}\text { Inclement Weather \& Atmospheric } \\
\text { Disturbances }\end{array}$ & 58 & 21.09 & 1390 & 19.35 \\
\hline Thunderstorms / Rain & 8 & 2.91 & 415 & 5.78 \\
\hline Wind Shear & 6 & 2.18 & 175 & 2.44 \\
\hline Turbulence & 5 & 1.82 & 90 & 1.25 \\
\hline Wake Vortex & 7 & 2.55 & 284 & 3.95 \\
\hline Snow / Icing & 32 & 11.64 & 426 & 5.93 \\
\hline Poor Visibility & 19 & 6.91 & 832 & 11.58 \\
\hline Fog / Haze & 8 & 2.91 & 300 & 4.18 \\
\hline Night & 11 & 4.00 & 532 & 7.40 \\
\hline Obstacle & 9 & 3.27 & 486 & 6.76 \\
\hline Fixed & 2 & 0.73 & 4 & 0.56 \\
\hline Moving & 7 & 2.55 & 482 & 6.71 \\
\hline
\end{tabular}

Diagrams of temporal sequences for each category are shown in Appendix B, Figures B-1 through B-6. The diagrams for sequences initiated with adverse onboard conditions are shown in Figs. B-1 through B-3. Fig. B-2 shows that a vehicle upset is the most common result of inappropriate crew action/inaction occurring in at least $71 \%$ of events (30 of 42). Furthermore, a vehicle upset is a direct result of inappropriate crew action in at least 22 of the 30 events. The highest number of fatalities occurred with a vehicle upset (53\%) following inappropriate crew response (34\%).

Similarly, Fig. B-1 shows that a vehicle upset occurs as a result of system and components failures in at least $64 \%$ of the events but is much less often as a direct result of the failure or malfunction but more commonly preceded by inappropriate crew action/inaction. Following system and component failures, vehicle upset is involved in $75 \%$ of fatalities. Similar results are shown by events initiated by vehicle impairment (shown in Fig. B-3) where vehicle upset occurs in at least $63 \%$ of the events, inappropriate crew action occurs in $54 \%$ of events and vehicle upset is involved in $70 \%$ of fatalities.

The diagrams for events initiated by external hazards and disturbances are shown in Fig. B-4 through B-6. Figure B-4 indicates that there is no clearly dominant factor immediately following inclement weather as the initiating precursor. However a vehicle upset occurs in 69\% (40 of 58) of events and inappropriate crew action in $60 \%$ (35 of 58) of events, similar to the discussion of adverse onboard conditions. 


\section{Preliminary Definition of Future Potential Risks}

Future potential risks relevant to LOC must be considered in developing a comprehensive set of test scenarios that enable forward-looking mitigation to emerging and future hazards. An initial set of future risks based on current trends is provided in Table 5, based on previous work (see Ref. 12).

Table 5. Potential future LOC risks list by trend.

\begin{tabular}{|c|l|l|}
\hline No. & \multicolumn{1}{|c|}{ Trend / Condition } & \multicolumn{1}{|c|}{ Potential LOC Risk Factors } \\
\hline 1 & Increased Automation without Improved Crew Interfaces & Increase in Inappropriate Crew Response \\
\hline 2 & $\begin{array}{l}\text { Future Vehicle Configurations without Identification of Upset } \\
\text { Characteristics }\end{array}$ & Increased Incidents of Vehicle Upsets \\
\hline 3 & $\begin{array}{l}\text { Increased System Complexity without Comprehensive } \\
\text { Evaluation Process }\end{array}$ & $\begin{array}{l}\text { Increase in System Faults / Failures / Errors / } \\
\text { Insufficiencies }\end{array}$ \\
\hline 4 & High-Density Operations in Terminal Area & Increase in Wake Vortex Encounters \\
\hline 5 & High-Density Operations in Terminal Area & Increase in Pilot Workload \\
\hline 6 & Increase in Flight Deck Automation & Decrease in Manual Piloting Skills \\
\hline 7 & All-Weather Operations & Increase in Snow/Icing Encounters \\
\hline 8 & All-Weather Operations in Terminal Area & Increase in Wind Shear / Turbulence Encounters \\
\hline 9 & High-Density Mixed-Vehicle Operations & $\begin{array}{l}\text { Increased Incidence of Near-Miss and Mid-Air } \\
\text { Collision Events }\end{array}$ \\
\hline 10 & $\begin{array}{l}\text { New Vehicle Materials with Lack of Long-Term Data on Aging } \\
\text { and Damage Tolerance }\end{array}$ & \begin{tabular}{l} 
Increase in Damage-Initiated LOC Events \\
\hline
\end{tabular} \\
\hline
\end{tabular}

\section{Future Research: Aircraft Loss-of-Control Test Scenarios}

Future research will include: 1.) finalizing the accident / incident set; 2.) performing an analysis of the accident / incident set using a team consensus process; 3.) defining future risks that utilize all available sources of information as determined by CAST, the FAA and ICAO, and others in the aviation community; and 4.) developing a comprehensive set of LOC test scenarios based on the final analysis of accidents, incidents and future potential risks. The test scenarios will include adverse vehicle conditions, inappropriate crew actions / inaction, external hazards and disturbances, and abnormal vehicle dynamics and upset conditions occurring as single precursors and combined as multiple precursor events. The test scenarios will also include recommended evaluation methods, and flight conditions. An example scenario format is given below in Table 6 based on previous work (see Ref. 12).

Table 6. Potential format for LOC test scenarios.

\begin{tabular}{|c|c|c|c|c|c|c|c|}
\hline $\begin{array}{l}\text { Scenario } \\
\text { Number }\end{array}$ & $\begin{array}{l}\text { Recommended } \\
\text { Evaluation } \\
\text { Methods }\end{array}$ & $\begin{array}{c}\text { Scenario } \\
\text { Description }\end{array}$ & $\begin{array}{c}\text { Flight } \\
\text { Condition }\end{array}$ & $\begin{array}{l}\text { Adverse Vehicle } \\
\text { Conditions }\end{array}$ & $\begin{array}{l}\text { Inappropriate Crew } \\
\text { Action / Inaction }\end{array}$ & $\begin{array}{l}\text { External Hazards \& } \\
\text { Disturbances }\end{array}$ & $\begin{array}{c}\text { Abnormal Vehicle } \\
\text { Dynamics I Vehicle Upset } \\
\text { Conditions }\end{array}$ \\
\hline \multicolumn{8}{|c|}{ Three Precursor LOC Scenarios: Vehicle Impairment $\rightarrow$ Vehicle Upset $\rightarrow$ Inappropriate Crew Response } \\
\hline 54 & $\begin{array}{l}\text { Analysis, Batch } \\
\text { Simulation, Piloted } \\
\text { Simulation }\end{array}$ & $\begin{array}{c}\text { Icing with } \\
\text { Vehicle } \\
\text { Impairment } \\
\text { Followed by } \\
\text { Vehicle Stall } \\
\text { and } \\
\text { Inappropriate } \\
\text { Crew } \\
\text { Response }\end{array}$ & $\begin{array}{l}\text { Approach, } \\
\text { Takeoff, } \\
\text { Cruise }\end{array}$ & $\begin{array}{l}\text { 1. Various Degrees of } \\
\text { Vehicle Dynamics } \\
\text { Changes under Airframe } \\
\text { Ice Accretion (from Mild to } \\
\text { Destabilizing), Varying } \\
\text { Degrees of Engine Icing } \\
\text { Effects from None to } \\
\text { Thrust Roll-back }\end{array}$ & $\begin{array}{l}\text { 3. Crew Responds } \\
\text { Inappropriately: } \\
\text { a. Delayed Reaction } \\
\text { b. Incorrect Recovery }\end{array}$ & $\begin{array}{l}\text { 1. Simulator: Icing Conditions } \\
\text { with and without Snow }\end{array}$ & $\begin{array}{l}\text { 2. Stall, Various Levels of De- } \\
\text { Stabilizing Effects from None } \\
\text { to Unstable in } \\
\text { a. One } \\
\text { b. Two } \\
\text { c. Three Axes }\end{array}$ \\
\hline \multicolumn{8}{|c|}{ Four Precursor LOC Scenarios: Vehicle Failure $\rightarrow$ Inappropriate Crew Response $\rightarrow>$ Upset $\rightarrow$ Vehicle Damage } \\
\hline 55 & $\begin{array}{l}\text { Analysis, Batch } \\
\text { Simulation, Piloted } \\
\text { Simulation }\end{array}$ & $\begin{array}{c}\text { Engine } \\
\text { Failure } \\
\text { Followed by } \\
\text { Crew } \\
\text { Distraction } \\
\text { Leading to } \\
\text { Upset and } \\
\text { Vehicle } \\
\text { Damage } \\
\end{array}$ & Cruise & $\begin{array}{l}\text { 1. Single Engine Failure } \\
\text { (100\% Thrust Loss); } \\
\text { 4. Various Levels of } \\
\text { Structural Damage with } \\
\text { and without Loss of } \\
\text { Control Effector }\end{array}$ & $\begin{array}{l}\text { 2. Crew Distraction } \\
\text { Resulting in Delayed } \\
\text { Response Followed by } \\
\text { Excessive Response }\end{array}$ & & $\begin{array}{l}\text { 3. Decreased Airspeed, } \\
\text { Asymmetric Forces / } \\
\text { Moments, Stall / Departure }\end{array}$ \\
\hline
\end{tabular}

A full set of test scenarios developed through team consensus will be published in a later paper, including a correlation of the scenarios to the accidents, incidents, and future risks defined in the final analysis results. 


\section{Conclusion}

This paper presents preliminary analysis results for a set of 275 accidents and incidents that occurred over the period 1996-2010 and involving aircraft at and above 12,500 lbs. Statistics for this set are provided in terms of each five-year period, aircraft type, operational type, and phase of flight. The analysis was performed by subsets allocated to the team, and consisted of determining individual precursor contributions to accidents and fatalities, identifying worst-case precursor combinations, and determining the temporal sequencing of precursors leading to LOC accidents and incidents from the set. Preliminary findings based on the results of this paper indicate that system failures or malfunctions, crew actions or inactions, vehicle impairment conditions, and vehicle upsets contribute the most to accidents and fatalities, followed by inclement weather or atmospheric disturbances and poor visibility. Individual precursors that contributed most to the accidents and fatalities in the analyzed set came from the System Failures / Malfunctions, Inappropriate Crew Action / Inaction, and Vehicle Upset sub-categories. Other key contributors included Vehicle Impairment, Inclement Weather and Atmospheric Disturbances, and Abnormal Vehicle Dynamics. Worst-case precursor combinations (in terms of number of accidents and fatalities) were dominated by System \& Component Failures / Malfunctions, Inappropriate Crew Action / Inaction, and Vehicle Upset Conditions, with contributions from the Weather \& Atmospheric Disturbance and Poor Visibility subcategories. The next worst-case combination involved Vehicle Impairment with and without Vehicle Upsets. Further examination of the worst-case combination of Inappropriate Crew Action / Inaction - Poor Visibility Vehicle Upset revealed that the underlying precursors consisted entirely of Loss of Attitude and Energy State Awareness, occurred predominantly at night, and led primarily to abnormal trajectories, uncontrolled descent, and stall/departure. Additional worst-case precursor-level evaluations will be performed for the final analysis. The preliminary worst-case analysis of FBW aircraft indicated that System Failures / Malfunctions and Vehicle Impairment combined with Vehicle Upsets were the largest contributors to accidents and fatalities. Analysis of temporal sequencing indicated that LOC events were usually precipitated by an adverse onboard condition, and most often by a system failure or malfunction, or by an external hazard or disturbance, usually due to weather or poor visibility. Vehicle upsets most often occurred as the second, third, or fourth factor in the LOC sequence indicating that multiple precursors can lead to an upset. Evaluation of sequence diagrams of Appendix B indicated that vehicle upset occurred as a result of system and components failures in at least $64 \%$ of the events evaluated, but was much less often as a direct result of the failure or malfunction but more commonly preceded by inappropriate crew action/inaction. Following system and component failures, vehicle upset was involved in $75 \%$ of fatalities. Similar results were shown by events initiated by vehicle impairment. Furthermore, a vehicle upset is a direct result of inappropriate crew action in at least 22 of the 30 events. The highest number of fatalities occurred with a vehicle upset (53\%) following inappropriate crew response (34\%). The diagrams for events initiated by external hazards and disturbances indicate that there is no clearly dominant factor immediately following inclement weather as the initiating precursor. However a vehicle upset occurs in 69\% (40 of 58) of events and inappropriate crew action in $60 \%$ (35 of 58) of events, similar to that previously discussed as a result of adverse onboard conditions.

Follow-on research will involve re-evaluating the accident / incident set using a consensus process to ensure consistency, defining a set of future LOC-related risks, and generating a comprehensive set of LOC test scenarios based on the final accident / incident analysis and future risks. The analysis results and test scenarios resulting from this research can be used in the development and evaluation of technology solutions, such as onboard systems, that provide improved LOC prevention and recovery capabilities. Wider application of this research to broader LOC solutions is also envisioned. 
Appendix A: Accident / Incident Set

\begin{tabular}{|c|c|c|c|c|c|c|c|c|c|c|c|c|c|}
\hline Date & Aircraft & Regist & Operat'n & Ident & Loc'n & Light & Weather & Fat & Grd & Dam & Phase & Result & Occurrence \\
\hline 1/7/1996 & DC-9 & N922VV & SCHED & VJA 558 & KBNA & Day & VMC & 0 & 0 & $\mathrm{~S}$ & Landing & Hard Landing & Uncomm Spoiler Ext \\
\hline 2/6/1996 & B-757 & TCGEN & NSCHD & ALW 301 & MDPP & Night & $\mathrm{N} / \mathrm{R}$ & 189 & 0 & $\mathrm{D}$ & Climb & Uncont Coll w/Terr & Instrument Failure \\
\hline 2/12/1996 & $\begin{array}{l}\text { GAF- } \\
\text { N24 }\end{array}$ & $\mathrm{N} 224 \mathrm{E}$ & SCHED & N224E & MTPP & Day & VMC & 10 & 0 & $\mathrm{D}$ & Initial climb & Uncont Coll w/Terr & Loss-of-Control (Vmc) \\
\hline 2/19/1996 & CE-550 & DCASH & EXEC & PWF SH & & $N / R$ & $N / R$ & 10 & 0 & $\mathrm{D}$ & Approach & Uncont Coll w/Terr & Icing Stall \\
\hline 2/22/1996 & MD-11 & & SCHED & CAL 4 & RCTP & $N / R$ & $\mathrm{~N} / \mathrm{R}$ & 0 & 0 & $N / R$ & Initial climb & Upset & Pilot Induced Osc (PIO) \\
\hline $5 / 11 / 1996$ & DC-9 & N904VJ & SCHED & VJA 592 & KMIA & Day & VMC & 110 & 0 & $\mathrm{D}$ & Climb & Uncont Coll w/Terr & Struct Fail - Fire/Expl \\
\hline 6/5/1996 & MD-80 & N224AA & SCHED & AAL 873 & KABQ & Day & VMC & 0 & 0 & $\mathrm{M}$ & Landg flare & Hard Landing & Atmos Disturbance \\
\hline 6/9/1996 & B-737 & N221US & SCHED & SGR 517 & KRIC & Night & VMC & 0 & 0 & $\mathrm{~N}$ & Approach & Upset & Uncommanded Bank \\
\hline 6/14/1996 & A-320 & N347NW & SCHED & NWA 395 & KBOS & Day & VMC & 0 & 0 & $\mathrm{~N}$ & Climb & Uncomm Pitch & Flight Control System \\
\hline 6/21/1996 & A-340 & DAIBE & SCHED & DLH 436 & KDFW & $N / R$ & $\mathrm{~N} / \mathrm{R}$ & 0 & 0 & $N / R$ & Climb & Cabin Injuries & Unexpected Cont Gains \\
\hline 7/13/1996 & MD-11 & N1768D & SCHED & AAL 8D & & Day & VMC & 0 & & $\mathrm{~N}$ & Descent & Cabin Injuries & Override Autopilot \\
\hline 7/17/1996 & B-747 & N93119 & SCHED & TWA 800 & KJFK & Dusk & VMC & 230 & 0 & $\mathrm{D}$ & Climb & Uncont Coll w/Terr & Struct Fail - Fire/Expl \\
\hline 7/20/1996 & DC-6A & N313RS & NSCHD & NAC 33 & PARS & Day & VMC & 4 & 0 & $\mathrm{D}$ & Cruise & Uncont Coll w/Terr & Struct Fail - Fire/Expl \\
\hline 10/22/1996 & B-707 & N751MA & NSCHD & MIRA MA & SEMT & $N / R$ & $\mathrm{~N} / \mathrm{R}$ & 4 & 23 & $\mathrm{D}$ & Climb & Coll w/Obstacle & Undetermined \\
\hline 10/31/1996 & FO-100 & PTMRK & SCHED & TAM 402 & SBSP & Day & $N / R$ & 96 & 3 & $\mathrm{D}$ & Initial climb & Coll w/Obstacle & Loss-of-Control (Vmc) \\
\hline 11/7/1996 & B-727 & 5NBBG & SCHED & ADK 86 & & $N / R$ & $N / R$ & 144 & 0 & $\mathrm{D}$ & Approach & Uncont Coll w/Terr & Aggressive Maneuver \\
\hline 11/12/1996 & Il-76TD & UN76435 & NSCHD & KZA 1907 & & $N / R$ & $N / R$ & 37 & 0 & $\mathrm{D}$ & Descent & Uncont Coll w/Terr & Struct Fail - Midair \\
\hline 11/12/1996 & B-747 & HZAIH & SCHED & SVA 763 & & $\mathrm{~N} / \mathrm{R}$ & $\mathrm{N} / \mathrm{R}$ & 312 & 0 & $\mathrm{D}$ & Climb & Uncont Coll w/Terr & Struct Fail - Midair \\
\hline 12/9/1996 & DC-3C & N75142 & NSCHD & D7T 42 & KBOI & Night & VMC & 2 & 0 & $\mathrm{D}$ & Initial climb & Collision w/Terrain & Stall \\
\hline 12/10/1996 & An-74 & RA74037 & NSCHD & VSA 37 & UERR & Night & $\mathrm{N} / \mathrm{R}$ & 0 & 0 & $\mathrm{D}$ & Initial climb & Collision w/Terrain & Thrust Rev-Unwanted \\
\hline $12 / 21 / 1996$ & An-32B & HK4008X & NSCHD & SDV $8 X$ & SKRG & Night & $N / R$ & 4 & 0 & $\mathrm{D}$ & Approach & Collision w/Terrain & Undetermined \\
\hline 1/9/1997 & E-120 & N265CA & SCHED & COM 3272 & KDTW & Day & IMC & 29 & 0 & $\mathrm{D}$ & Descent & Uncont Coll w/Terr & Autopilot-Induced Stall \\
\hline $1 / 25 / 1997$ & Il-76 & RA76834 & NREV & VSO 34 & UHMA & $\mathrm{N} / \mathrm{R}$ & $\mathrm{N} / \mathrm{R}$ & 0 & 0 & $\mathrm{D}$ & Initial climb & Collision w/Terrain & TO w/Incorrect Config \\
\hline 2/1/1997 & HS-748 & 6VAEO & NSCHD & DS EO & GOTT & $\mathrm{N} / \mathrm{R}$ & $\mathrm{N} / \mathrm{R}$ & 23 & 0 & $\mathrm{D}$ & Initial climb & Collision w/Terrain & Undetermined \\
\hline 3/2/1997 & BE-200 & N117WM & EXEC & PVT WM & KSLC & Dusk & IMC & 1 & 0 & $\mathrm{~S}$ & Final appr & Uncont Coll w/Terr & Stall \\
\hline 3/14/1997 & $\mathrm{F}-27$ & D2TFP & NSCHD & DTA FP & FCBB & $\mathrm{N} / \mathrm{R}$ & $\mathrm{N} / \mathrm{R}$ & 3 & 0 & $\mathrm{D}$ & Initial climb & Collision w/Terrain & Undetermined \\
\hline 4/14/1997 & An-24 & RA46516 & NSCHD & RA46516 & & Day & $N / R$ & 50 & 0 & $\mathrm{D}$ & Cruise & Uncont Coll w/Terr & Struct Fail - Fatigue \\
\hline 4/19/1997 & BA-АТP & PKMTX & SCHED & MNA 106 & WIOD & Night & $N / R$ & 15 & 0 & $\mathrm{D}$ & Approach & Uncont Coll w/Terr & Atmosph Disturbance \\
\hline 5/8/1997 & B-737 & B2925 & SCHED & CSN 3456 & ZGSZ & Night & IMC & 35 & 0 & $\mathrm{D}$ & Landing & Hard Landing & Atmosph Disturbance \\
\hline $5 / 12 / 1997$ & A-300 & N90070 & SCHED & AAL 903 & KPBI & Day & IMC & 0 & 0 & $\mathrm{M}$ & Level off & Upset & Stall \\
\hline 5/20/1997 & AC-1121 & N1121F & EXEC & PVT 1F & & Day & IMC & 4 & 0 & $\mathrm{D}$ & Cruise & Uncont Coll w/Terr & Atmosph Disturbance \\
\hline 6/1/1997 & MD-11 & JA8580 & SCHED & JAL 706 & RJNA & Day & $\mathrm{N} / \mathrm{R}$ & 0 & 0 & $\mathrm{M}$ & Descent & Unknown & Override Autopilot \\
\hline 7/3/1997 & $\mathrm{F}-27$ & VTSSA & NSCHD & LBE SA & VABB & Night & IMC & 2 & 0 & $\mathrm{D}$ & Initial climb & Uncont Coll w/Terr & Undetermined \\
\hline 7/12/1997 & DC-9 & N9138 & SCHED & NWA 944 & KMEM & Day & VMC & 0 & 0 & $\mathrm{M} / \mathrm{N}$ & Landing & Upset & Uncommanded Bank \\
\hline 8/7/1997 & DC-8 & N27UA & NSCHD & FBF 101 & KMIA & Day & VMC & 4 & 1 & D & Initial climb & Uncont Coll w/Terr & Load/c/g Out Of Range \\
\hline 10/10/1997 & DC-9 & LVWEG & SCHED & AUT 2553 & & Night & IMC & 74 & 0 & $\mathrm{D}$ & Descent & Uncont Coll w/Terr & Struct Fail - Exc Limit \\
\hline 12/13/1997 & SA-226 & СР1635 & NSCHD & SAVE 35 & SLVT & $\mathrm{N} / \mathrm{R}$ & $\mathrm{N} / \mathrm{R}$ & 10 & 0 & $\mathrm{D}$ & Initial climb & Collision w/Terrain & Undetermined \\
\hline $12 / 16 / 1997$ & CL-600 & CFSKI & SCHED & ACA 646 & CYFC & Night & IMC & 0 & 0 & $\mathrm{D}$ & Go-around & Collision w/Terrain & Stall \\
\hline 2/16/1998 & A-300 & B1814 & SCHED & CAL 676 & RCTP & Night & IMC & 196 & 7 & $\mathrm{D}$ & Approach & Uncont Coll w/Terr & Stall \\
\hline 3/18/1998 & SF-340 & B12255 & SCHED & FOS 55 & RCPO & Night & IMC & 13 & 0 & $\mathrm{D}$ & Climb & Uncont Coll w/Terr & Spatial Disorientation \\
\hline 5/21/1998 & DC-10 & N68043 & SCHED & COA 75 & KLAX & Day & VMC & 0 & 0 & $\mathrm{M}$ & Climb & Uncomm Pitch & Autopilot \\
\hline 6/18/1998 & SA-226 & CGQAL & SCHED & PRO 420 & CYUL & Day & $\mathrm{N} / \mathrm{R}$ & 11 & 0 & $\mathrm{D}$ & Climb & Uncont Coll w/Terr & Struct Fail - Fire/Expl \\
\hline 7/23/1998 & An-12 & RA11886 & NSCHD & RA11886 & ULLP & Day & $\mathrm{N} / \mathrm{R}$ & 0 & 0 & $\mathrm{D}$ & Takeoff roll & Collision w/Terrain & Loss-of-Control (Vmc) \\
\hline 7/28/1998 & SA-227 & ECFXD & NSCHD & SWT 704 & LEBL & Night & VMC & 2 & 0 & $\mathrm{D}$ & Approach & Uncont Coll w/Terr & Loss-of-Control (Vmc) \\
\hline
\end{tabular}

American Institute of Aeronautics and Astronautics 


\begin{tabular}{|c|c|c|c|c|c|c|c|c|c|c|c|c|c|}
\hline Date & Aircraft & Regist & Operat'n & Ident & Loc'n & Light & Weather & Fat & Grd & Dam & Phase & Result & Occurrence \\
\hline 7/30/1998 & Do-228 & VTEJW & SCHED & LLR JW & VOCC & Day & VMC & 6 & 3 & $\mathrm{D}$ & Initial climb & Uncont Coll w/Terr & Flight Control Actuator \\
\hline 7/30/1998 & BE-1900 & FGSJM & SCHED & PRB 706 & & Day & VMC & 14 & 0 & $\mathrm{D}$ & Approach & Uncont Coll w/Terr & Struct Fail - Midair \\
\hline 8/24/1998 & DC-3 & ZSNKK & NSCHD & SPZ KK & FAWB & Day & VMC & 1 & 0 & $\mathrm{D}$ & Initial climb & Uncont Coll w/Terr & TO w/Mis-set Trim \\
\hline 9/2/1998 & MD-11 & HBIWF & SCHED & SWR 111 & CYHZ & Night & $\mathrm{N} / \mathrm{R}$ & 229 & 0 & $\mathrm{D}$ & Cruise & Collision w/Terrain & Loss Of All Att Displ \\
\hline 10/17/1998 & BE-99 & N299GL & SCHED & AIP 5010 & KMSO & Night & VMC & 0 & 0 & $\mathrm{~s}$ & Go-around & Collision w/Terrain & Fail To Maint Airspeed \\
\hline 10/18/1998 & A-320 & EITLI & SCHED & TRZ LI & EIDW & $\mathrm{N} / \mathrm{R}$ & N/R & 0 & 0 & $\mathrm{M} / \mathrm{N}$ & Approach & Upset & Jammed Flight Controls \\
\hline $10 / 21 / 1998$ & E-120 & PTWKH & NSCHD & PTWKH & SBFZ & Day & $\mathrm{N} / \mathrm{R}$ & 3 & 1 & $\mathrm{D}$ & VFR pattern & Uncont Coll w/Terr & Improper Contr Oper’n \\
\hline 11/11/1998 & SF-340 & VHLPI & SCHED & KDA PI & YMML & Day & IMC & 0 & 0 & $\mathrm{~N}$ & holding & Upset & Icing Stall \\
\hline $12 / 2 / 1998$ & CE-501 & N501EZ & NREV & EXEC EZ & & Day & VMC & 1 & 0 & $\mathrm{D}$ & Cruise & Uncont Coll w/Terr & Undetermined \\
\hline 12/4/1998 & An-12 & LZSFG & NSCHD & LXR FG & LPLA & Night & $\mathrm{N} / \mathrm{R}$ & 7 & 0 & $\mathrm{D}$ & Initial climb & Uncont Coll w/Terr & Asymm Thrust/Drag \\
\hline $12 / 11 / 1998$ & A-310 & HSTIA & SCHED & TIA 261 & VSSB & Night & IMC & 101 & 0 & $\mathrm{D}$ & Approach & Uncont Coll w/Terr & Somatogravic Illusion \\
\hline 1/12/1999 & $\mathrm{F}-27$ & GCHNL & NSCHD & EXS NL & EGJB & $\mathrm{N} / \mathrm{R}$ & $\mathrm{N} / \mathrm{R}$ & 2 & 0 & $\mathrm{D}$ & Approach & Uncont Coll w/Terr & Stall \\
\hline $1 / 28 / 1999$ & LR-35 & $\mathrm{N} 130 \mathrm{~F}$ & NSCHD & USC 251 & KMDW & Night & VMC & 0 & 0 & $\mathrm{~S}$ & Landing & Hard Landing & Unstabilized Approach \\
\hline 2/2/1999 & An-12 & EYASS & NSCHD & FDN SS & FNLU & Night & $\mathrm{N} / \mathrm{R}$ & 11 & 19 & $\mathrm{D}$ & Initial climb & Coll w/Obstacle & Undetermined \\
\hline 2/24/1999 & Tu-154 & B2622 & SCHED & CSW 4509 & ZSWZ & $\mathrm{N} / \mathrm{R}$ & N/R & 61 & 0 & $\mathrm{D}$ & Approach & Uncont Coll w/Terr & Flight Cont Disconnect \\
\hline 4/5/1999 & DHC-6 & N838MA & NSCHD & DCC MA & KLNA & Day & VMC & 0 & 0 & $\mathrm{~S}$ & Approach & Collision w/Terrain & Loss-of-Control (Vmc) \\
\hline 4/7/1999 & B-737 & TCJEP & NREV & THY 5904 & LTAF & Night & IMC & 6 & 0 & $\mathrm{D}$ & Climb & Uncont Coll w/Terr & Instrument Failure \\
\hline 4/15/1999 & MD-11 & HL7373 & SCHED & KAL 6316 & ZSSS & Day & $\mathrm{N} / \mathrm{R}$ & 3 & 5 & $\mathrm{D}$ & Climb & Uncont Coll w/Terr & Spatial Disorientation \\
\hline 8/26/1999 & Yak-40 & UK87848 & SCHED & UZB 48 & UTNT & Day & $N / R$ & 2 & 0 & $\mathrm{D}$ & Go-around & Coll w/Obstacle & Undetermined \\
\hline 8/31/1999 & B-737 & LVWRZ & SCHED & LPR 3142 & SABE & Night & N/R & 63 & 2 & $\mathrm{D}$ & Initial climb & Uncont Coll w/Terr & TO w/Incorrect Config \\
\hline 9/2/1999 & B-737 & N371UA & SCHED & UAL 2036 & & Day & VMC & 0 & 0 & M & Cruise & Atmos Disturbance & Wake Turbulence \\
\hline 9/14/1999 & DA-900 & SXECH & EXEC & OAL 3838 & LROP & $\mathrm{N} / \mathrm{R}$ & N/R & 7 & 0 & $\mathrm{~S}$ & Descent & Upset & Pilot Induced Osc (PIO) \\
\hline 9/24/1999 & A-320 & CFKCO & SCHED & ACA 630 & CYSJ & Night & VMC & 0 & 0 & M & Landing & Landed Short & Flt Contr Mode Change \\
\hline 10/9/1999 & DA-900 & N523AC & EXEC & PVT AC & KGRR & $\mathrm{N} / \mathrm{R}$ & $\mathrm{N} / \mathrm{R}$ & 0 & 0 & & Descent & Aircraft Oscillat'ns & Override Autopilot \\
\hline 10/18/1999 & SF-340 & SELES & SCHED & GAO 750 & ENSN & Night & IMC & 0 & 0 & $\mathrm{M} / \mathrm{N}$ & Climb & Upset & Autopilot-Induced Stall \\
\hline 10/25/1999 & LR-35 & N47BA & NSCHD & SJ8 BA & & $\mathrm{N} / \mathrm{R}$ & $\mathrm{N} / \mathrm{R}$ & 6 & 0 & $\mathrm{D}$ & Climb & Spiral Dive & Incapacitation: Hypoxia \\
\hline 11/9/1999 & DC-9 & XATKN & SCHED & TEJ 725 & MMPN & Night & $N / R$ & 18 & 0 & $\mathrm{D}$ & Climb & Collision w/Terrain & Spatial Disorientation \\
\hline $12 / 5 / 1999$ & Il-114 & UK91004 & NSCHD & СТВ 04 & UUDD & $\mathrm{N} / \mathrm{R}$ & N/R & 5 & 0 & $\mathrm{D}$ & Initial climb & Coll w/Obstacle & Jammed Flight Controls \\
\hline 12/12/1999 & IAI-1124 & N50PL & EXEC & PVT PL & & Day & VMC & 3 & 0 & $\mathrm{D}$ & Descent & Coll w/Obstacle & Flight Control Disconn \\
\hline $12 / 22 / 1999$ & B-747 & HL7451 & SCHED & KAL 8509 & EGSS & Night & $\mathrm{N} / \mathrm{R}$ & 4 & 0 & $\mathrm{D}$ & Climb & Uncont Coll w/Terr & Instrument Failure \\
\hline $1 / 5 / 2000$ & E-110 & 5NAXL & SCHED & EAN XL & DNAA & $\mathrm{N} / \mathrm{R}$ & $\mathrm{N} / \mathrm{R}$ & 1 & 1 & $\mathrm{D}$ & Approach & Collision w/Terrain & Stall \\
\hline $1 / 10 / 2000$ & SF-340 & HBAKK & SCHED & SWR 498 & LSZH & Night & IMC & 10 & 0 & $\mathrm{D}$ & Initial climb & Spiral Dive & Somatogravic Illusion \\
\hline $1 / 30 / 2000$ & A-310 & 5YBEN & SCHED & KQA 431 & DIAP & Night & VMC & 169 & 0 & $\mathrm{D}$ & Initial climb & Collision w/Terrain & Stall \\
\hline $1 / 31 / 2000$ & MD-80 & N963AS & SCHED & ASA 261 & & Day & VMC & 88 & 0 & $\mathrm{D}$ & Cruise & Uncont Coll w/Terr & Jammed Flight Controls \\
\hline $2 / 16 / 2000$ & DC-8 & N8079U & NSCHD & EWW 17 & KMHR & Night & VMC & 3 & 0 & $\mathrm{D}$ & Initial climb & Uncont Coll w/Terr & Flight Control Disconn \\
\hline $2 / 27 / 2000$ & B-747 & GBDXL & SCHED & BAW 179 & & Night & IMC & 0 & 0 & $\mathrm{~N}$ & Descent & Upset & Uncommanded Pitch \\
\hline $3 / 9 / 2000$ & Yak-40 & RA88170 & NSCHD & VGV 70 & UUEE & Day & $\mathrm{N} / \mathrm{R}$ & 9 & 0 & $\mathrm{D}$ & Initial climb & Uncont Coll w/Terr & TO w/Contam Wing \\
\hline $3 / 17 / 2000$ & DC-3 & CFNTF & NSCHD & PTSN TF & CYJC & $\mathrm{N} / \mathrm{R}$ & N/R & 2 & 0 & $\mathrm{D}$ & Go-around & Uncont Coll w/Terr & Load-c/g Out Of Rng \\
\hline 3/30/2000 & B-767 & N182DN & SCHED & DAL 106 & KJFK & Night & IMC & 0 & 0 & $\mathrm{M}$ & Climb & Upset & Spatial Disorientation \\
\hline $5 / 2 / 2000$ & LR-35 & GMURI & NSCHD & NEX RI & LFLL & $\mathrm{N} / \mathrm{R}$ & N/R & 2 & 0 & $\mathrm{D}$ & Landing & Uncont Coll w/Terr & Loss-of-Control (Vmc) \\
\hline $5 / 21 / 2000$ & JS-3101 & N16EJ & NSCHD & ORA EJ & KAVP & Day & IMC & 19 & 0 & $\mathrm{D}$ & Approach & Uncont Coll w/Terr & Loss-of-Control (Vmc) \\
\hline $6 / 22 / 2000$ & Y-7 & B3479 & SCHED & CWU 343 & ZHHH & Day & IMC & 42 & 7 & $\mathrm{D}$ & Approach & Coll w/Obstacle & Wind Shear \\
\hline $6 / 23 / 2000$ & LR-55 & N220JC & NREV & UJT JC & KBCT & Day & VMC & 3 & 0 & $\mathrm{D}$ & Climb & Uncont Coll w/Terr & Struct Fail - Midair \\
\hline $6 / 27 / 2000$ & A-300 & N14065 & SCHED & AAL 65 & EGLL & $N / R$ & N/R & 0 & 0 & $\mathrm{~N}$ & Climb & Diversion & Wake Turbulence \\
\hline $7 / 17 / 2000$ & B-737 & VTEGD & SCHED & LLR 7412 & VEPT & Day & MVMC & 55 & 5 & $\mathrm{D}$ & Approach & Uncont Coll w/Terr & Stall \\
\hline $7 / 19 / 2000$ & G-159 & CGNAK & NSCHD & AWV 9807 & & Night & IMC & 2 & 0 & $\mathrm{D}$ & Cruise & Uncont Coll w/Terr & Loss-of-Control (Vmc) \\
\hline $7 / 20 / 2000$ & DC-3 & N54AA & NSCHD & N54AA & MYNN & Day & VMC & 2 & 0 & $\mathrm{D}$ & Initial climb & Collision w/Terrain & Undetermined \\
\hline $7 / 25 / 2000$ & AS-100 & FBTSC & NSCHD & AFR 4590 & LFPG & Day & VMC & 109 & 4 & $\mathrm{D}$ & Takeoff & Uncont Coll w/Terr & Struct Fail - Fire/Expl \\
\hline $8 / 23 / 2000$ & A-320 & A4OEK & SCHED & GFA 72 & OBBI & Night & VMC & 143 & 0 & $\mathrm{D}$ & Missed appr & Collision w/Terrain & Somatogravic Illusion \\
\hline
\end{tabular}

American Institute of Aeronautics and Astronautics 


\begin{tabular}{|c|c|c|c|c|c|c|c|c|c|c|c|c|c|}
\hline Date & Aircraft & Regist & Operat'n & Ident & Loc'n & Light & Weather & Fat & Grd & Dam & Phase & Result & Occurrence \\
\hline $8 / 31 / 2000$ & An-26 & D2FDI & NSCHD & NCL DI & FNSA & $\mathrm{N} / \mathrm{R}$ & $\mathrm{N} / \mathrm{R}$ & 44 & 0 & $\mathrm{D}$ & Cruise & Uncont Coll w/Terr & Undetermined \\
\hline $10 / 2 / 2000$ & A-340 & TCJDN & SCHED & THY DN & & $N / R$ & N/R & 0 & 0 & $\mathrm{~N}$ & Cruise & Loss Of Separation & Flt Cont Mode Change \\
\hline $10 / 26 / 2000$ & CL-600 & N958CA & SCHED & COM CA & & Day & VMC & 0 & 0 & $\mathrm{~N}$ & Cruise & Uncont Coll w/Terr & Wake Turbulence \\
\hline $11 / 1 / 2000$ & DHC-6 & CGGAW & SCHED & YWZ 151 & & Day & $\mathrm{N} / \mathrm{R}$ & 0 & 0 & $\mathrm{D}$ & Initial climb & Collision w/Terrain & Loss-of-Control (Vmc) \\
\hline $11 / 9 / 2000$ & SA-226 & N731AC & NSCHD & ETA4 1000 & KFWA & Night & IMC & 1 & 0 & $\mathrm{D}$ & Initial climb & Uncont Coll w/Terr & Instrument Failure \\
\hline $11 / 15 / 2000$ & An-24 & D2FCG & NSCHD & API CG & FNLU & Day & $\mathrm{N} / \mathrm{R}$ & 57 & 0 & $\mathrm{D}$ & Initial climb & Collision w/Terrain & Loss-of-Control (Vmc) \\
\hline $11 / 25 / 2000$ & MD-11 & N582FE & SCHED & FDE 3015 & KEWR & Day & VMC & 0 & 0 & $\mathrm{~N}$ & Climb & Pilot Induced Osc & Flight Controls \\
\hline $12 / 2 / 2000$ & LR-35 & CGDJH & NSCHD & CGDJH & CYVR & $N / R$ & $\mathrm{~N} / \mathrm{R}$ & 0 & 0 & $\mathrm{~N}$ & Climb & Uncomm Bank & Jammed Flight Controls \\
\hline $12 / 27 / 2000$ & E-135 & N721HS & SCHED & EGF 230 & KORD & Night & VMC & 0 & 0 & $\mathrm{~N}$ & Initial climb & Upset & Jammed Flight Controls \\
\hline $1 / 25 / 2001$ & DC-3 & YV224C & NSCHD & RUC 225 & SVCB & Day & $\mathrm{N} / \mathrm{R}$ & 24 & 0 & $\mathrm{D}$ & VFR pattern & Uncont Coll w/Terr & Loss-of-Control (Vmc) \\
\hline $1 / 27 / 2001$ & BE-200 & N81PF & EXEC & JEK PF & & Dusk & IMC & 10 & 0 & $\mathrm{D}$ & Cruise & Loss-of-Control & Instrument Failure \\
\hline 2/7/2001 & A-320 & ECHKJ & SCHED & IBE 1456 & LEBB & Night & VMC & 0 & 0 & $\mathrm{D}$ & Landing & Hard Landing & Unexpect Control Gains \\
\hline 2/8/2001 & LR-35 & IMOCO & NREV & IMOCO & EDDN & Day & VMC & 3 & 0 & $\mathrm{D}$ & Approach & Uncont Coll w/Terr & Stall \\
\hline $3 / 17 / 2001$ & A-320 & N357NW & SCHED & NWA 985 & KDTW & Night & IMC & 0 & 0 & $\mathrm{~S}$ & Rotation & $\begin{array}{l}\text { Crash After } \\
\text { Takeoff }\end{array}$ & Pilot Induced Osc (PIO) \\
\hline 3/19/2001 & E-120 & N266CA & SCHED & COM 5054 & KРBA & Day & IMC & 0 & 0 & $\mathrm{~S}$ & Descent & Upset & Icing Stall \\
\hline $3 / 20 / 2001$ & A-320 & DAIPW & SCHED & DLH PW & EDFF & $N / R$ & $\mathrm{~N} / \mathrm{R}$ & 0 & 0 & $\mathrm{~N}$ & Initial climb & Uncomm Bank & Reversed Controls \\
\hline $3 / 24 / 2001$ & DHC-6 & FOGES & SCHED & ISB 1501 & TFFJ & Day & VMC & 19 & 1 & $\mathrm{D}$ & VFR pattern & Uncont Coll w/Terr & Loss-of-Control (Vmc) \\
\hline $4 / 2 / 2001$ & CE-501 & N405PC & NREV & N405PC & KGRB & Day & IMC & 1 & 0 & $\mathrm{D}$ & Climb & Coll w/Obstacle & Spatial Disorientation \\
\hline $5 / 25 / 2001$ & A-340 & FGLZC & SCHED & AFR 3682 & SOCA & Day & VMC & 0 & 0 & $\mathrm{M}$ & Landing & Landed Short & Atmosph Disturbance \\
\hline $7 / 4 / 2001$ & Tu-154 & RA85845 & SCHED & VLK 352 & & Night & IMC & 145 & 0 & $\mathrm{D}$ & Approach & Uncont Coll w/Terr & Autopilot-Induced Stall \\
\hline 8/9/2001 & BE-200 & N899RW & EXEC & EXEC RW & KOKZ & Day & IMC & 0 & 0 & $\mathrm{D}$ & Circling appr & Collision w/Terrain & Stall \\
\hline $8 / 24 / 2001$ & LR-25 & N153TW & NSCHD & AJI TW & KITH & Night & IMC & 2 & 0 & $\mathrm{D}$ & Initial climb & Collision w/Terrain & Somatogravic Illusion \\
\hline $9 / 12 / 2001$ & Let-410 & ХААСM & NSCHD & ХАACM & MMCT & Day & VMC & 19 & 0 & $\mathrm{D}$ & Initial climb & Uncont Coll w/Terr & Fail To Maintain Cont \\
\hline $9 / 14 / 2001$ & BE-1900 & CGSKC & NSCHD & SKK 621 & CYYT & Night & IMC & 0 & 0 & $\mathrm{D}$ & Initial climb & Forced Landing & Uncommanded Pitch \\
\hline $9 / 18 / 2001$ & Let-410 & TGCFE & SCHED & TGCFE & MGGT & $\mathrm{N} / \mathrm{R}$ & $\mathrm{N} / \mathrm{R}$ & 8 & 0 & $\mathrm{D}$ & Initial climb & Uncont Coll w/Terr & Stall \\
\hline $10 / 4 / 2001$ & Tu-154 & RA85693 & SCHED & SBI 1812 & & Day & $\mathrm{N} / \mathrm{R}$ & 78 & 0 & $\mathrm{D}$ & Cruise & Uncont Coll w/Terr & Hostile Action \\
\hline $10 / 10 / 2001$ & SA-226 & ECGDV & NSCHD & FTL 101 & & Day & IMC & 10 & 0 & $\mathrm{D}$ & Cruise & Uncont Coll w/Terr & Loss Of All Att Displ \\
\hline $10 / 16 / 2001$ & E-145 & N825MJ & SCHED & ASH 5733 & KROA & Night & VMC & 0 & 0 & $\mathrm{~S}$ & Landing & Hard Landing & Stall \\
\hline $11 / 12 / 2001$ & A-300 & N14053 & SCHED & AAL 587 & KJFK & Day & VMC & 260 & 5 & $\mathrm{D}$ & Climb & In-flight Breakup & Wake Turbulence \\
\hline $11 / 19 / 2001$ & Il-18 & RA75840 & NSCHD & LDF 40 & & $\mathrm{~N} / \mathrm{R}$ & $\mathrm{N} / \mathrm{R}$ & 27 & 0 & $\mathrm{D}$ & Cruise & Uncont Coll w/Terr & Flight Control System \\
\hline $11 / 22 / 2001$ & LR-25 & N5UJ & NREV & UJT UJ & KPIT & Day & VMC & 2 & 0 & $\mathrm{D}$ & Initial climb & Collision w/Terrain & Overcontrol \\
\hline $12 / 10 / 2001$ & LR-24 & N997TD & NREV & X5CA 36 & & Night & VMC & 2 & 0 & $\mathrm{D}$ & Descent & Uncont Coll w/Terr & Undetermined \\
\hline $12 / 14 / 2001$ & DC-8 & N825BX & NSCHD & RTI 8101 & PANC & Night & VMC & 0 & 0 & $\mathrm{~N}$ & Initial climb & Uncomm Bank & Flight Control Hardover \\
\hline $12 / 20 / 2001$ & CE-560 & HBVLV & NREV & EGU 220 & LSZH & Night & IMC & 2 & 0 & $\mathrm{D}$ & Initial climb & Uncont Coll w/Terr & Somatogravic Illusion \\
\hline $1 / 4 / 2002$ & CL-600 & N90AG & NSCHD & PVT AG & EGBB & Day & VMC & 5 & 0 & $\mathrm{D}$ & Initial climb & Uncont Coll w/Terr & TO w/Contam Wing \\
\hline $1 / 22 / 2002$ & B-757 & TFFIO & SCHED & ICE 315 & ENGM & Day & IMC & 0 & 0 & $\mathrm{~N}$ & Go-around & Upset & Somatogravic Illusion \\
\hline $4 / 12 / 2002$ & SA-227 & ECGKR & NSCHD & TDC KR & LEPA & Night & N/R & 2 & 0 & $\mathrm{D}$ & Approach & Collision w/Terrain & Aggressive Maneuver \\
\hline $5 / 4 / 2002$ & BAC-111 & 5NESF & SCHED & EXW 4226 & KNKN & Day & $N / R$ & 71 & 78 & $\mathrm{D}$ & Cruise & Uncont Coll w/Terr & Stall \\
\hline $5 / 25 / 2002$ & B-747 & B18255 & SCHED & CAL 611 & & Day & $\mathrm{N} / \mathrm{R}$ & 225 & 0 & $\mathrm{D}$ & Cruise & Uncont Coll w/Terr & Struct Fail - Fatigue \\
\hline $6 / 3 / 2002$ & MD-11 & N588FE & NSCHD & FEX 5181 & & Night & IMC & 0 & 0 & $\mathrm{~S}$ & Descent & Structural Failure & Overcontrol \\
\hline $6 / 4 / 2002$ & MD-80 & N823NK & SCHED & NKS 970 & & Day & VMC & 0 & 0 & $\mathrm{~N}$ & Cruise & Upset & Autopilot-Induced Stall \\
\hline $6 / 14 / 2002$ & A-330 & CGHLM & SCHED & ACA 875 & EDDF & $\mathrm{N} / \mathrm{R}$ & $\mathrm{N} / \mathrm{R}$ & 0 & 0 & $\mathrm{~N}$ & Approach & Uncomm Pitch & Flight Control Logic \\
\hline $6 / 28 / 2002$ & SF-340 & VHOLM & SCHED & HZL 185 & YBTH & Night & $\mathrm{N} / \mathrm{R}$ & 0 & 0 & $\mathrm{~N}$ & Circling appr & Upset & Autopilot-Induced Stall \\
\hline 7/1/2002 & B-757 & A9CDHL & NSCHD & DHL 611 & & Night & $N / R$ & 2 & 0 & $\mathrm{D}$ & Cruise & Uncont Coll w/Terr & Struct Fail - Midair \\
\hline $7 / 1 / 2002$ & Tu-154 & RA85816 & NSCHD & BTC 2937 & & Night & $N / R$ & 69 & 0 & $\mathrm{D}$ & Cruise & Uncont Coll w/Terr & Struct Fail - Midair \\
\hline $7 / 28 / 2002$ & Il-86 & RA86060 & NREV & PLK 60 & UUEE & Day & $N / R$ & 14 & 0 & $\mathrm{D}$ & Initial climb & Uncont Coll w/Terr & Runaway Pitch Trim \\
\hline $8 / 14 / 2002$ & ATR-42 & PTMTS & NSCHD & TTL 5561 & & Night & $\mathrm{N} / \mathrm{R}$ & 2 & 0 & $\mathrm{D}$ & Cruise & Uncont Coll w/Terr & Runaway Pitch Trim \\
\hline $10 / 9 / 2002$ & B-747 & N661US & SCHED & NWA 85 & PANC & Night & VMC & 0 & 0 & $\mathrm{M}$ & Cruise & Uncomm Bank & Flight Control Hardover \\
\hline
\end{tabular}

American Institute of Aeronautics and Astronautics 


\begin{tabular}{|c|c|c|c|c|c|c|c|c|c|c|c|c|c|}
\hline Date & Aircraft & Regist & Operat'n & Ident & Loc'n & Light & Weather & Fat & Grd & Dam & Phase & Result & Occurrence \\
\hline $10 / 20 / 2002$ & B-757 & TFFII & SCHED & ICE 662 & KBWI & Night & $\mathrm{N} / \mathrm{R}$ & 0 & 0 & $\mathrm{~N}$ & Climb & Upset & Spatial Disorientation \\
\hline $11 / 8 / 2002$ & IAI-1124 & N61RS & NREV & BQVA RS & KSKX & Day & VMC & 2 & 0 & $\mathrm{D}$ & Approach & Uncont Coll w/Terr & Atmosph Disturbance \\
\hline $12 / 3 / 2002$ & A-300 & Unknown & SCHED & Unknown & EDDM & Day & $\mathrm{N} / \mathrm{R}$ & 0 & 0 & $\mathrm{~N}$ & Climb & Exceeded Vne/Vmo & Controls (Trim) \\
\hline $12 / 7 / 2002$ & A-320 & CGIUF & SCHED & ACA 1130 & CYYZ & $N / R$ & $N / R$ & 0 & 0 & $\mathrm{~N}$ & Final appr & Go Around & Pilot Induced Osc (PIO) \\
\hline $12 / 7 / 2002$ & A-320 & CGJVX & SCHED & ACA 457 & CYYZ & $\mathrm{N} / \mathrm{R}$ & $\mathrm{N} / \mathrm{R}$ & 0 & 0 & $\mathrm{~N}$ & Final appr & Hard Landing & Pilot Induced Osc (PIO) \\
\hline $12 / 21 / 2002$ & ATR-72 & B22708 & NSCHD & TNA 791 & & Night & IMC & 2 & 0 & $\mathrm{D}$ & Descent & Uncont Coll w/Terr & Autopilot-Induced Stall \\
\hline $12 / 27 / 2002$ & Let-410 & 9XRRB & SCHED & 9XRRB & FMCV & Day & IMC & 1 & 0 & $\mathrm{D}$ & Missed appr & Uncont Coll w/Terr & Instrument Failure \\
\hline $1 / 8 / 2003$ & BE-1900 & N233YV & SCHED & AMW 5481 & KCLT & Day & VMC & 21 & 0 & $\mathrm{D}$ & Initial climb & Uncont Coll w/Terr & Flt Cont Integrity Lost \\
\hline $2 / 10 / 2003$ & An-28 & ESNOY & SCHED & ENI 827 & EETN & Night & IMC & 2 & 0 & $\mathrm{D}$ & Initial climb & Coll w/Obstacle & TO w/Contam Wing \\
\hline 3/6/2003 & B-737 & 7TVEZ & SCHED & DAH 6289 & DAAT & Day & $\mathrm{N} / \mathrm{R}$ & 102 & 0 & $\mathrm{D}$ & Initial climb & Uncont Coll w/Terr & Stall \\
\hline $4 / 23 / 2003$ & BE-99 & CFDYF & SCHED & ABS YF & CYPA & Day & $\mathrm{N} / \mathrm{R}$ & 0 & 0 & $\mathrm{D}$ & Approach & Uncont Coll w/Terr & Flight Control Actuator \\
\hline $5 / 1 / 2003$ & LR-45 & IERJC & NREV & IERJC & ASN & $N / R$ & $N / R$ & 2 & 0 & $\mathrm{D}$ & Initial climb & Uncont Coll w/Terr & Struct Fail - Birdstrike \\
\hline $6 / 16 / 2003$ & A-320 & CGTDK & NSCHD & SSV DK & EGGD & $N / R$ & $N / R$ & 0 & 0 & $\mathrm{~S}$ & Landg flare & Hard Landing & Unexpect Contr Gains \\
\hline $7 / 8 / 2003$ & B-737 & STAFK & SCHED & SUD 139 & HSSP & Night & $\mathrm{N} / \mathrm{R}$ & 116 & 0 & $\mathrm{D}$ & Missed appr & Uncont Coll w/Terr & Fail To Maintain Cont \\
\hline $8 / 4 / 2003$ & LR-35 & $\mathrm{N} 135 \mathrm{PT}$ & NREV & RM6A PT & KGON & Day & VMC & 2 & 0 & $\mathrm{D}$ & Approach & Coll w/Obstacle & Inadvert Control Input \\
\hline $8 / 24 / 2003$ & Let-410 & HHPRV & SCHED & HHPRV & MTCH & Night & $\mathrm{N} / \mathrm{R}$ & 21 & 0 & $\mathrm{D}$ & VFR pattern & Uncont Coll w/Terr & Fail To Maintain Cont \\
\hline $8 / 26 / 2003$ & BE-1900 & N240CJ & NREV & CJC 9446 & KHYA & Day & VMC & 2 & 0 & $\mathrm{D}$ & Initial climb & Uncont Coll w/Terr & Reversed Controls \\
\hline $10 / 3 / 2003$ & CV-580 & ZKKFU & SCHED & AFN 642 & & Night & IMC & 2 & 0 & $\mathrm{D}$ & Descent & In-flight Breakup & Icing Stall \\
\hline $10 / 26 / 2003$ & FH-227 & LVMGV & NSCHD & CTZ 760 & & Night & $\mathrm{N} / \mathrm{R}$ & 5 & 0 & $\mathrm{D}$ & Cruise & Uncont Coll w/Terr & Loss-of-Control (Vmc) \\
\hline $11 / 22 / 2003$ & A-300 & OODLL & NSCHD & BCS LL & ORBS & Day & VMC & 0 & 0 & $\mathrm{~S}$ & Climb & Runway Departure & Hostile Action \\
\hline $12 / 23 / 2003$ & LR-24 & N600XJ & EXEC & PVT XJ & & Day & VMC & 2 & 0 & $\mathrm{D}$ & Climb & Uncont Coll w/Terr & Undetermined \\
\hline $1 / 3 / 2004$ & B-737 & SUZCF & NSCHD & FLS 604 & HESH & Night & VMC & 148 & 0 & $\mathrm{D}$ & Climb & Spiral Dive & Spatial Disorientation \\
\hline $2 / 10 / 2004$ & FO-50 & EPLCA & SCHED & IRK 7170 & OMSI & Day & $\mathrm{N} / \mathrm{R}$ & 43 & 0 & $\mathrm{D}$ & Final appr & Uncont Coll w/Terr & Undetermined \\
\hline $3 / 4 / 2004$ & Il-76 & URZVA & NSCHD & AZV VA & UBBB & $\mathrm{N} / \mathrm{R}$ & $\mathrm{N} / \mathrm{R}$ & 3 & 0 & $\mathrm{D}$ & Initial climb & Uncont Coll w/Terr & TO w/Incorrect Config \\
\hline $3 / 19 / 2004$ & LR-35 & N800AW & NSCHD & BSYA AW & KUCA & $N / R$ & IMC & 0 & 0 & $\mathrm{~S}$ & Go-around & Hard Landing & Stall \\
\hline $5 / 5 / 2004$ & SA-227 & HK4275X & EXEC & PVT 5X & SKLC & Day & $\mathrm{N} / \mathrm{R}$ & 5 & 0 & $\mathrm{D}$ & Final appr & Uncont Coll w/Terr & Stall \\
\hline $5 / 6 / 2004$ & Let-410 & 9XREF & NSCHD & 9XREF & & Day & $N / R$ & 6 & 0 & $\mathrm{D}$ & Takeoff & Uncont Coll w/Terr & Stall \\
\hline $5 / 17 / 2004$ & DHC-6 & 8QTMC & SCHED & TMW MC & VRMM & Day & $N / R$ & 0 & 0 & $\mathrm{D}$ & Initial climb & Coll w/Obstacle & TO w/Incorrect Config \\
\hline $5 / 18 / 2004$ & Il-76 & 4KAZ27 & NSCHD & AHC 27 & ZWWW & Day & $\mathrm{N} / \mathrm{R}$ & 7 & 0 & $\mathrm{D}$ & Initial climb & Collision w/Terrain & Undetermined \\
\hline $6 / 18 / 2004$ & SF-340 & VHKEQ & SCHED & REX EQ & YMML & Day & IMC & 0 & 0 & & Descent & Upset & Autopilot-Induced Stall \\
\hline $7 / 2 / 2004$ & IAI-1124 & N280AT & NSCHD & N280AT & МРТО & Day & $\mathrm{N} / \mathrm{R}$ & 6 & 0 & $\mathrm{D}$ & Initial climb & Uncont Coll w/Terr & Undetermined \\
\hline $7 / 21 / 2004$ & DC-9 & XABCS & SCHED & SER 706 & MMMX & $N / R$ & $N / R$ & 0 & 0 & $\mathrm{D}$ & Initial climb & Collision w/Terrain & Wind Shear \\
\hline $8 / 11 / 2004$ & B-737 & 3XGCM & SCHED & GIB CM & GFLL & $\mathrm{N} / \mathrm{R}$ & $N / R$ & 0 & 0 & $\mathrm{D}$ & Initial climb & Collision w/Terrain & TO w/Incorrect Config \\
\hline $10 / 5 / 2004$ & An-12 & STSAF & NSCHD & SRW AF & & Day & $N / R$ & 4 & 0 & $\mathrm{D}$ & Cruise & Uncont Coll w/Terr & Fail To Maintain Cont \\
\hline $10 / 14 / 2004$ & B-747 & 9GMKJ & NSCHD & MKA 1602 & CYHZ & Night & $\mathrm{N} / \mathrm{R}$ & 7 & 0 & $\mathrm{D}$ & Initial climb & Collision w/Terrain & Stall \\
\hline $10 / 14 / 2004$ & CL-600 & N8396A & NREV & FLG 3701 & KJEF & Night & VMC & 2 & 0 & $\mathrm{D}$ & Cruise & Collision w/Terrain & Autopilot-Induced Stall \\
\hline $11 / 21 / 2004$ & CL-600 & B3072 & SCHED & CES 5210 & ZBOW & Day & $\mathrm{N} / \mathrm{R}$ & 53 & 2 & $\mathrm{D}$ & Initial climb & Uncont Coll w/Terr & TO w/Contam Wing \\
\hline $11 / 28 / 2004$ & CL-600 & N873G & NSCHD & YQCA 73 & KMTJ & Day & IMC & 3 & 0 & $\mathrm{D}$ & Initial climb & Uncont Coll w/Terr & TO w/Contam Wing \\
\hline $11 / 30 / 2004$ & HFB-320 & N604GA & NREV & GAE GA & KSUS & Night & IMC & 2 & 0 & $\mathrm{D}$ & Initial climb & Collision w/Terrain & Controls (Trim) \\
\hline $12 / 10 / 2004$ & BE-200 & N648KA & NSCHD & YSDA KA & TS94 & Day & VMC & 0 & 0 & $\mathrm{D}$ & Initial climb & Coll w/Obstacle & Stall \\
\hline $1 / 13 / 2005$ & E-110 & N49BA & NSCHD & RLR 2352 & KEEN & Night & IMC & 1 & 0 & $\mathrm{D}$ & Missed appr & Uncont Coll w/Terr & Loss-of-Control (Vmc) \\
\hline 2/16/2005 & CE-560 & N500AT & EXEC & N500AT & KPUB & Day & IMC & 8 & 0 & $\mathrm{D}$ & Final appr & Uncont Coll w/Terr & Icing Stall \\
\hline 2/24/2005 & IAI-1124 & XCCOL & EXEC & PVT OL & & Day & $\mathrm{N} / \mathrm{R}$ & 7 & 0 & $\mathrm{D}$ & Approach & Collision w/Terrain & Undetermined \\
\hline 3/15/2005 & An-26 & OB1778P & NSCHD & AMP 8P & SPIM & Day & $N / R$ & 0 & 0 & $\mathrm{~S}$ & Initial climb & Collision w/Terrain & TO w/Incorrect Config \\
\hline 3/26/2005 & Let-410 & HK4146 & SCHED & WCW 9955 & SKPV & Day & $\mathrm{N} / \mathrm{R}$ & 9 & 0 & $\mathrm{D}$ & Initial climb & Uncont Coll w/Terr & Loss-of-Control (Vmc) \\
\hline $5 / 2 / 2005$ & SA-227 & ZKPOA & SCHED & AWK 23 & & Night & IMC & 2 & 0 & $\mathrm{D}$ & Cruise & Uncont Coll w/Terr & Load-c/g Out Of Range \\
\hline $5 / 12 / 2005$ & MD-90 & N910ME & SCHED & MEP 490 & KIRK & Night & IMC & 0 & 0 & $\mathrm{~N}$ & Cruise & Aircraft Oscillat'ns & Instrument Failure \\
\hline $5 / 21 / 2005$ & CL-600 & N699CW & NREV & DGFA CW & KAGS & Night & VMC & 0 & 0 & $\mathrm{~N}$ & Climb & Cabin Injuries & Aggressive Maneuver \\
\hline $5 / 27 / 2005$ & DHC-8 & CGZKH & SCHED & CGZKH & CYYT & $\mathrm{N} / \mathrm{R}$ & $\mathrm{N} / \mathrm{R}$ & 0 & 0 & $\mathrm{~N}$ & Climb & Upset & Autopilot-Induced Stall \\
\hline
\end{tabular}

American Institute of Aeronautics and Astronautics 


\begin{tabular}{|c|c|c|c|c|c|c|c|c|c|c|c|c|c|}
\hline Date & Aircraft & Regist & Operat'n & Ident & Loc'n & Light & Weather & Fat & Grd & Dam & Phase & Result & Occurrence \\
\hline 8/1/2005 & B-777 & 9MMRG & SCHED & MAS 124 & YPРH & $\mathrm{N} / \mathrm{R}$ & $\mathrm{N} / \mathrm{R}$ & 0 & 0 & $\mathrm{~N}$ & Climb & Upset & Uncommanded Pitch \\
\hline $8 / 14 / 2005$ & B-737 & 5BDBY & SCHED & HCY 522 & LGAV & N/R & $N / R$ & 121 & 0 & $\mathrm{D}$ & Climb & Uncont Coll w/Terr & Incapacitation: Hypoxia \\
\hline 8/16/2005 & MD-80 & HK4374X & NSCHD & WCW 708 & & Night & $N / R$ & 160 & 0 & $\mathrm{D}$ & Cruise & Uncont Coll w/Terr & Autopilot-Induced Stall \\
\hline 9/5/2005 & B-737 & PRBRY & SCHED & BRB 907 & & $\mathrm{~N} / \mathrm{R}$ & $N / R$ & 0 & 0 & $N / R$ & Cruise & Upset & Uncommanded Bank \\
\hline 9/5/2005 & B-737 & PKRIM & SCHED & MDL 91 & WIMM & $N / R$ & N/R & 100 & 49 & $\mathrm{D}$ & Initial climb & Coll w/Obstacle & TO w/Incorrect Config \\
\hline $10 / 3 / 2005$ & E-170 & N650RW & SCHED & UHL 7621 & KIAD & Day & VMC & 0 & 0 & $\mathrm{~N}$ & VFR pattern & Cabin Injuries & Aggressive Maneuver \\
\hline 10/22/2005 & B-737 & 5NBFN & SCHED & BVU 210 & & Night & $\mathrm{N} / \mathrm{R}$ & 117 & 0 & D & Climb & Collision w/Terrain & Undetermined \\
\hline 11/8/2005 & E-110 & N7801Q & NSCHD & BQTA 352 & КMHT & Day & VMC & 0 & 0 & $\mathrm{D}$ & Initial climb & Coll w/Obstacle & Loss-of-Control (Vmc) \\
\hline 12/19/2005 & G-73 & N2969 & SCHED & CHK 101 & КMPB & Day & VMC & 20 & 0 & $\mathrm{D}$ & Initial climb & Uncont Coll w/Terr & Struct Fail - Fatigue \\
\hline $12 / 23 / 2005$ & An-140 & 4KAZ48 & SCHED & AHY 217 & & Night & IMC & 23 & 0 & $\mathrm{D}$ & Cruise & Uncont Coll w/Terr & Loss Of All Att Displ \\
\hline $12 / 28 / 2005$ & LR-35 & N781RS & NREV & S2KA RS & KTRK & Day & IMC & 2 & 0 & $\mathrm{D}$ & Approach & Uncont Coll w/Terr & Stall \\
\hline $1 / 2 / 2006$ & SF-340 & N390AE & SCHED & SIM 3008 & & Day & IMC & 0 & 0 & $\mathrm{~N}$ & Climb & Upset & Autopilot-Induced Stall \\
\hline $1 / 5 / 2006$ & CE-560 & N391QS & NSCHD & DXTA QS & KARV & Day & VMC & 0 & & $\mathrm{~S}$ & Landing & Coll w/Obstacle & Stall \\
\hline 2/8/2006 & SA-226 & N629EK & NSCHD & GAE EK & & Day & VMC & 1 & 0 & $\mathrm{D}$ & Cruise & Uncont Coll w/Terr & Undetermined \\
\hline 2/9/2006 & CL-600 & N900LG & EXEC & PVT LG & KASE & Day & VMC & 0 & 0 & $\mathrm{~S}$ & Approach & Hard Landing & Wake Turbulence \\
\hline $5 / 3 / 2006$ & A-320 & EK32009 & SCHED & RNV 967 & URSS & Night & IMC & 113 & 0 & $\mathrm{D}$ & Missed appr & Uncont Coll w/Terr & Spatial Disorientation \\
\hline 6/21/2006 & DHC-6 & 9NAEQ & NSCHD & NYT EQ & VNJL & $\mathrm{N} / \mathrm{R}$ & $\mathrm{N} / \mathrm{R}$ & 9 & 0 & $\mathrm{D}$ & Go-around & Collision w/Terrain & Aggressive Maneuver \\
\hline 7/10/2006 & F-27 & APBAL & SCHED & PIA 688 & OPMT & Day & $N / R$ & 45 & 0 & $\mathrm{D}$ & Initial climb & Collision w/Terrain & Stall \\
\hline 8/13/2006 & L-387 & 7TVHG & NSCHD & DAH 2208 & & $N / R$ & $N / R$ & 3 & 0 & $\mathrm{D}$ & Cruise & Uncont Coll w/Terr & Undetermined \\
\hline $8 / 22 / 2006$ & Tu-154 & RA85185 & SCHED & PLK 612 & & Day & IMC & 170 & 0 & $\mathrm{D}$ & Cruise & Uncont Coll w/Terr & Turbulence \\
\hline 9/29/2006 & B-737 & PRGTD & SCHED & GLO 1907 & & Day & VMC & 154 & 0 & $\mathrm{D}$ & Cruise & Uncont Coll w/Terr & Struct Fail - Midair \\
\hline 9/29/2006 & E-135 & N600XL & NREV & N600XL & SBCC & Day & VMC & 0 & 0 & $\mathrm{~S}$ & Cruise & Forced Landing & Struct Fail - Midair \\
\hline $10 / 23 / 2006$ & A-320 & N924FR & SCHED & FFT 539 & KDEN & Day & VMC & 0 & 0 & $\mathrm{~N}$ & Landg flare & Uncomm Pitch & Inadvertent Cont Input \\
\hline $10 / 29 / 2006$ & B-737 & 5NBFK & SCHED & ADK 53 & DNAA & Day & IMC & 96 & & $\mathrm{D}$ & Initial climb & Uncont Coll w/Terr & Wind Shear \\
\hline 10/31/2006 & CL-600 & N322FX & NREV & N322FX & KTEB & $N / R$ & VMC & 0 & & $\mathrm{~N}$ & Approach & Cabin Injuries & Aggressive Maneuver \\
\hline $11 / 30 / 2006$ & NA-265 & XATNP & NSCHD & FCS NP & MMCL & $N / R$ & $\mathrm{~N} / \mathrm{R}$ & 2 & 0 & $\mathrm{D}$ & Maneuvering & Coll w/Obstacle & Undetermined \\
\hline 1/1/2007 & B-737 & PKKKW & SCHED & DHI 574 & & Day & $\mathrm{N} / \mathrm{R}$ & 102 & 0 & $\mathrm{D}$ & Cruise & Spiral Dive & Spatial Disorientation \\
\hline $1 / 10 / 2007$ & LR-35 & N40AN & NSCHD & N40AN & КСMH & Night & VMC & 0 & 0 & $\mathrm{~S}$ & Maneuvering & Overstress & Fail To Maintain Cont \\
\hline $1 / 12 / 2007$ & CE-525 & N77215 & NREV & SQ6R 15 & KVNY & Day & VMC & 2 & 0 & $\mathrm{D}$ & Initial climb & Uncont Coll w/Terr & Stall \\
\hline $1 / 25 / 2007$ & FO-100 & FGMPG & SCHED & RAE 7775 & LFBP & Day & VMC & 0 & 1 & $\mathrm{~S}$ & Initial climb & Coll w/Obstacle & TO w/Contam Wing \\
\hline 2/13/2007 & CL-600 & N168CK & NREV & N168CK & UUWW & $N / R$ & IMC & 0 & 0 & $\mathrm{D}$ & Initial climb & Uncont Coll w/Terr & Undetermined \\
\hline 3/17/2007 & CE-500 & N511AT & NSCHD & N511AT & KBVY & Day & IMC & 0 & 0 & $\mathrm{~S}$ & Landg flare & Collision w/Terrain & Contam Airfoil \\
\hline 3/27/2007 & E-170 & HZAEN & SCHED & SVA 1866 & OERK & $\mathrm{N} / \mathrm{R}$ & N/R & 0 & 0 & $N / R$ & Descent & Uncomm Pitch & Undetermined \\
\hline $5 / 5 / 2007$ & B-737 & 5YKYA & SCHED & KQA 507 & FKKD & Night & $N / R$ & 114 & 0 & $\mathrm{D}$ & Initial climb & Spiral Dive & Spatial Disorientation \\
\hline $5 / 17 / 2007$ & Let-410 & TNAHE & NSCHD & SAFE HE & & $\mathrm{N} / \mathrm{R}$ & $\mathrm{N} / \mathrm{R}$ & 3 & 0 & $\mathrm{D}$ & Initial climb & Coll w/Obstacle & Undetermined \\
\hline 6/4/2007 & CE-550 & N500BP & NSCHD & DJQ BP & KMKE & Day & VMC & 6 & 0 & $\mathrm{D}$ & Climb & Spiral Dive & Spatial Disorientation \\
\hline 7/29/2007 & An-12 & RA93912 & NSCHD & VAS 9655 & UUDD & Night & $\mathrm{N} / \mathrm{R}$ & 7 & 0 & $\mathrm{D}$ & Initial climb & Uncont Coll w/Terr & Loss-of-Control (Vmc) \\
\hline 8/9/2007 & DHC-6 & FOIQI & SCHED & ТАH 1121 & NTTM & Day & VMC & 20 & 0 & $\mathrm{D}$ & Initial climb & Uncont Coll w/Terr & Flt Cont Integrity Lost \\
\hline 9/23/2007 & B-737 & GTHOF & SCHED & TOM OF & & Night & IMC & 0 & 0 & $\mathrm{~N}$ & Landing & Upset & Stall \\
\hline 10/17/2007 & LR-35 & N31MC & EXEC & PVT MC & KGLD & Day & IMC & 0 & & $\mathrm{~S}$ & Landing & Collision w/Terrain & Aircraft Pitch/Roll Osc \\
\hline $11 / 4 / 2007$ & LR-35 & PTOVC & NREV & PTOVC & SBMT & $\mathrm{N} / \mathrm{R}$ & N/R & 2 & 6 & $\mathrm{D}$ & Initial climb & Uncont Coll w/Terr & Undetermined \\
\hline $12 / 10 / 2007$ & BE-200 & N925TT & EXEC & PVT TT & KSMN & Dawn & IMC & 2 & & $\mathrm{D}$ & Initial climb & Coll w/Obstacle & TO w/Contam Wing \\
\hline $12 / 16 / 2007$ & CL-600 & N470ZW & SCHED & AWI 3758 & KPVD & Day & IMC & 0 & 0 & $\mathrm{~S}$ & Landing & Hard Landing & Stall \\
\hline $1 / 10 / 2008$ & A-320 & CGBHZ & SCHED & ACA 190 & KOMK & Night & N/R & 0 & 0 & M & Climb & Upset & Wake Turbulence \\
\hline $2 / 14 / 2008$ & CL-600 & EW101PJ & SCHED & BRU 1834 & UDYZ & N/R & $\mathrm{N} / \mathrm{R}$ & 0 & 0 & $\mathrm{D}$ & Initial climb & Collision w/Terrain & TO w/Contam Wing \\
\hline $3 / 4 / 2008$ & CE-500 & N113SH & NSCHD & N113SH & KPWA & Day & $\mathrm{N} / \mathrm{R}$ & 5 & 0 & $\mathrm{D}$ & Climb & Collision w/Terrain & Struct Fail - Birdstrike \\
\hline 4/9/2008 & SA-227 & VHOZA & NSCHD & VHOZA & YSSY & Night & VMC & 1 & 0 & $\mathrm{D}$ & Climb & Spiral Dive & Spatial Disorientation \\
\hline $5 / 23 / 2008$ & BE-1900 & N195GA & NSCHD & TIM 5008 & KBIL & Night & IMC & 1 & 0 & $\mathrm{D}$ & Initial climb & Uncont Coll w/Terr & Undetermined \\
\hline $5 / 26 / 2008$ & An-12 & RA12957 & NREV & GAI 2063 & USCC & $\mathrm{N} / \mathrm{R}$ & N/R & 9 & 0 & $\mathrm{D}$ & Climb & Uncont Coll w/Terr & Flt Cont Integrity Lost \\
\hline
\end{tabular}

American Institute of Aeronautics and Astronautics 


\begin{tabular}{|c|c|c|c|c|c|c|c|c|c|c|c|c|c|}
\hline Date & Aircraft & Regist & Operat'n & Ident & Loc'n & Light & Weather & Fat & Grd & Dam & Phase & Result & Occurrence \\
\hline $6 / 14 / 2008$ & MD-10 & N554FE & SCHED & FDE 764 & & $\mathrm{~N} / \mathrm{R}$ & VMC & 0 & & $\mathrm{~S}$ & holding & Exc Design Loads & Stall \\
\hline $6 / 18 / 2008$ & DHC-6 & N656WA & SCHED & WIG 6601 & KHYA & Day & VMC & 1 & 0 & $\mathrm{D}$ & Takeoff & Uncont Coll w/Terr & TO w/Gust Locks Eng \\
\hline $6 / 30 / 2008$ & Il-76 & STWTB & NSCHD & BBE 700 & HSSS & Day & $\mathrm{N} / \mathrm{R}$ & 4 & 0 & $\mathrm{D}$ & Initial climb & Collision w/Terrain & TO w/Incorrect Config \\
\hline $7 / 10 / 2008$ & BE-99 & CCCFM & SCHED & CCCFM & SCPF & $N / R$ & $\mathrm{~N} / \mathrm{R}$ & 9 & 0 & $\mathrm{D}$ & Initial climb & Uncont Coll w/Terr & Stall \\
\hline $7 / 16 / 2008$ & DHC-6 & CGBEB & NSCHD & NWI EB & & Day & VMC & 0 & 0 & $\mathrm{~s}$ & VFR pattern & Coll w/Obstacle & Stall \\
\hline $8 / 20 / 2008$ & MD-80 & ECHFP & SCHED & JKK 5022 & LEMD & $\mathrm{N} / \mathrm{R}$ & $\mathrm{N} / \mathrm{R}$ & 154 & 0 & $\mathrm{D}$ & Takeoff & Collision w/Terrain & TO w/Incorrect Config \\
\hline $9 / 14 / 2008$ & B-737 & VPBKO & SCHED & AFL KO & USPP & Night & IMC & 88 & 0 & $\mathrm{D}$ & Approach & Spiral Dive & Spatial Disorientation \\
\hline $10 / 7 / 2008$ & A-330 & VHQPA & SCHED & QFA 72 & YPLM & $\mathrm{N} / \mathrm{R}$ & $\mathrm{N} / \mathrm{R}$ & 0 & 0 & $\mathrm{M}$ & Cruise & Upset & Flight Control Logic \\
\hline $11 / 1 / 2008$ & C-212 & N437RA & NSCHD & ATS RA & & Dusk & VMC & 0 & 0 & $\mathrm{~S}$ & VFR pattern & Collision w/Terrain & Asymm Thrust/Drag \\
\hline $11 / 4 / 2008$ & LR-45 & XCVMC & EXEC & PVT MC & MMMX & $\mathrm{N} / \mathrm{R}$ & $\mathrm{N} / \mathrm{R}$ & 9 & 7 & & Approach & Uncont Coll w/Terr & Wake Turbulence \\
\hline $12 / 7 / 2008$ & LR-23 & XCLGD & EXEC & XCLGD & & $\mathrm{N} / \mathrm{R}$ & $N / R$ & 2 & 0 & $\mathrm{D}$ & Go-around & Uncont Coll w/Terr & Undetermined \\
\hline $1 / 27 / 2009$ & ATR-42 & N902FX & SCHED & CFS 8284 & KLLB & Night & IMC & 0 & 0 & $\mathrm{~s}$ & Final appr & Uncont Coll w/Terr & Stall \\
\hline 2/7/2009 & E-110 & PTSEA & NSCHD & PTSEA & SWKO & Day & $\mathrm{N} / \mathrm{R}$ & 24 & & $\mathrm{D}$ & Climb & Collision w/Terrain & Undetermined \\
\hline $2 / 7 / 2009$ & CE-650 & IFEEV & NREV & AOE 301 & & $\mathrm{~N} / \mathrm{R}$ & $\mathrm{N} / \mathrm{R}$ & 2 & 0 & $\mathrm{D}$ & Climb & Spiral Dive & Undetermined \\
\hline 2/12/2009 & DHC-8 & N200WQ & SCHED & CJC 3407 & KBUF & Night & VMC & 49 & 1 & $\mathrm{D}$ & Approach & Uncont Coll w/Terr & Stall \\
\hline $2 / 25 / 2009$ & B-737 & TCJGE & SCHED & THY 1951 & EHAM & Day & $\mathrm{N} / \mathrm{R}$ & 9 & 0 & $\mathrm{D}$ & Approach & Collision w/Terrain & Stall \\
\hline $5 / 11 / 2009$ & B-747 & GBYGA & SCHED & BAW GA & FAJS & Night & VMC & 0 & 0 & $\mathrm{~N}$ & Initial climb & Stall Buffet & Uncomm Config Chng \\
\hline $6 / 1 / 2009$ & A-330 & FGZCP & SCHED & AFR 447 & TASIL & Night & IMC & 228 & 0 & $\mathrm{D}$ & Cruise & Uncont Coll w/Terr & Spatial Disorientation \\
\hline 6/30/2009 & A-310 & 300 & SCHED & IYE 626 & FMCH & Night & VMC & 152 & 0 & $\mathrm{D}$ & Circling appr & Collision w/Terrain & Fail To Maintain Airspd \\
\hline $7 / 15 / 2009$ & Tu-154 & EPCPG & SCHED & CMP 7908 & & $\mathrm{~N} / \mathrm{R}$ & $\mathrm{N} / \mathrm{R}$ & 168 & 0 & $\mathrm{D}$ & Cruise & Collision w/Terrain & Undetermined \\
\hline 10/21/2009 & B-707 & STAKW & NSCHD & SUD 2241 & OMSJ & $N / R$ & $N / R$ & 6 & 0 & $\mathrm{D}$ & Initial climb & Uncont Coll w/Terr & Fail To Maintain Cont \\
\hline $11 / 1 / 2009$ & Il-76 & RF76801 & NREV & RF76801 & UERR & $N / R$ & $\mathrm{~N} / \mathrm{R}$ & 11 & 0 & $\mathrm{D}$ & Initial climb & Collision w/Terrain & Undetermined \\
\hline $11 / 28 / 2009$ & MD-11 & ZBAV & SCHED & SMJ 324 & ZSPD & $\mathrm{N} / \mathrm{R}$ & $\mathrm{N} / \mathrm{R}$ & 3 & 0 & $\mathrm{D}$ & Initial climb & Uncont Coll w/Terr & Undetermined \\
\hline $1 / 5 / 2010$ & LR-35 & N720RA & NREV & RAX 988 & KPWK & Day & VMC & 2 & & $\mathrm{D}$ & VFR pattern & Uncont Coll w/Terr & Undetermined \\
\hline $1 / 6 / 2010$ & BE-99 & N206AV & SCHED & JIKA AV & KEAR & Dawn & IMC & 0 & 0 & $\mathrm{~s}$ & Landg flare & Hard Landing & Icing Stall \\
\hline $1 / 21 / 2010$ & BE-1900 & N112AX & SCHED & AER 22 & PASD & Night & VMC & 2 & 0 & $\mathrm{D}$ & Initial climb & Uncont Coll w/Terr & Undetermined \\
\hline $1 / 25 / 2010$ & B-737 & ETANB & SCHED & ETH 409 & OLBA & Night & IMC & 90 & 0 & $\mathrm{D}$ & Climb & Spiral Dive & Spatial Disorientation \\
\hline 2/13/2010 & B-737 & N221WN & SCHED & SWA 2534 & KBUR & Day & VMC & 0 & & $\mathrm{~N}$ & Approach & Cabin Injuries & Aggressive Maneuver \\
\hline $2 / 14 / 2010$ & CE-550 & OKACH & NREV & TIE 039C & & Night & VMC & 2 & 0 & $\mathrm{D}$ & Cruise & Uncont Coll w/Terr & Intentional Acrobatics \\
\hline $5 / 12 / 2010$ & A-330 & 5AONG & SCHED & AAW 771 & HLLT & Day & IMC & 103 & 0 & $\mathrm{D}$ & Approach & Collision w/Terrain & Somatogravic Illusion \\
\hline $8 / 25 / 2010$ & Let- 410 & 9QCCN & SCHED & 9QCCN & ZFBO & N/R & $\mathrm{N} / \mathrm{R}$ & 20 & 0 & $\mathrm{D}$ & Final appr & Collision w/Terrain & Load - c/g Out Of Rng \\
\hline 9/3/2010 & B-747 & N571UP & SCHED & UPS UP & OMDB & Night & $N / R$ & 2 & 0 & $\mathrm{D}$ & Climb & Uncont Coll w/Terr & Struct Fail - Fire/Expl \\
\hline $10 / 11 / 2010$ & A-380 & FHPJA & SCHED & AFR 006 & KJFK & Day & $\mathrm{N} / \mathrm{R}$ & & 0 & $\mathrm{~N}$ & Final appr & Altitude Deviation & Flap/Slat Ext Spd Exc \\
\hline $11 / 4 / 2010$ & ATR-72 & CUT1549 & SCHED & CRN 883 & & $\mathrm{~N} / \mathrm{R}$ & IMC & 68 & 0 & $\mathrm{D}$ & Cruise & Uncont Coll w/Terr & Contaminated Airfoil \\
\hline $11 / 5 / 2010$ & BE-1900 & APBJD & NSCHD & JSJ JD & OPKC & $N / R$ & $\mathrm{~N} / \mathrm{R}$ & 21 & 0 & $\mathrm{D}$ & Initial climb & Collision w/Terrain & Loss-of-Control (Vmc) \\
\hline $11 / 28 / 2010$ & Il-76 & 4LGNI & NSCHD & 4LGNI & OPKC & $N / R$ & $\mathrm{~N} / \mathrm{R}$ & 8 & 4 & $\mathrm{D}$ & Initial climb & Coll w/Obstacle & Loss-of-Control (Vmc) \\
\hline
\end{tabular}

25

American Institute of Aeronautics and Astronautics 
Appendix B: Accident Sequence Diagrams

Events Fatalities

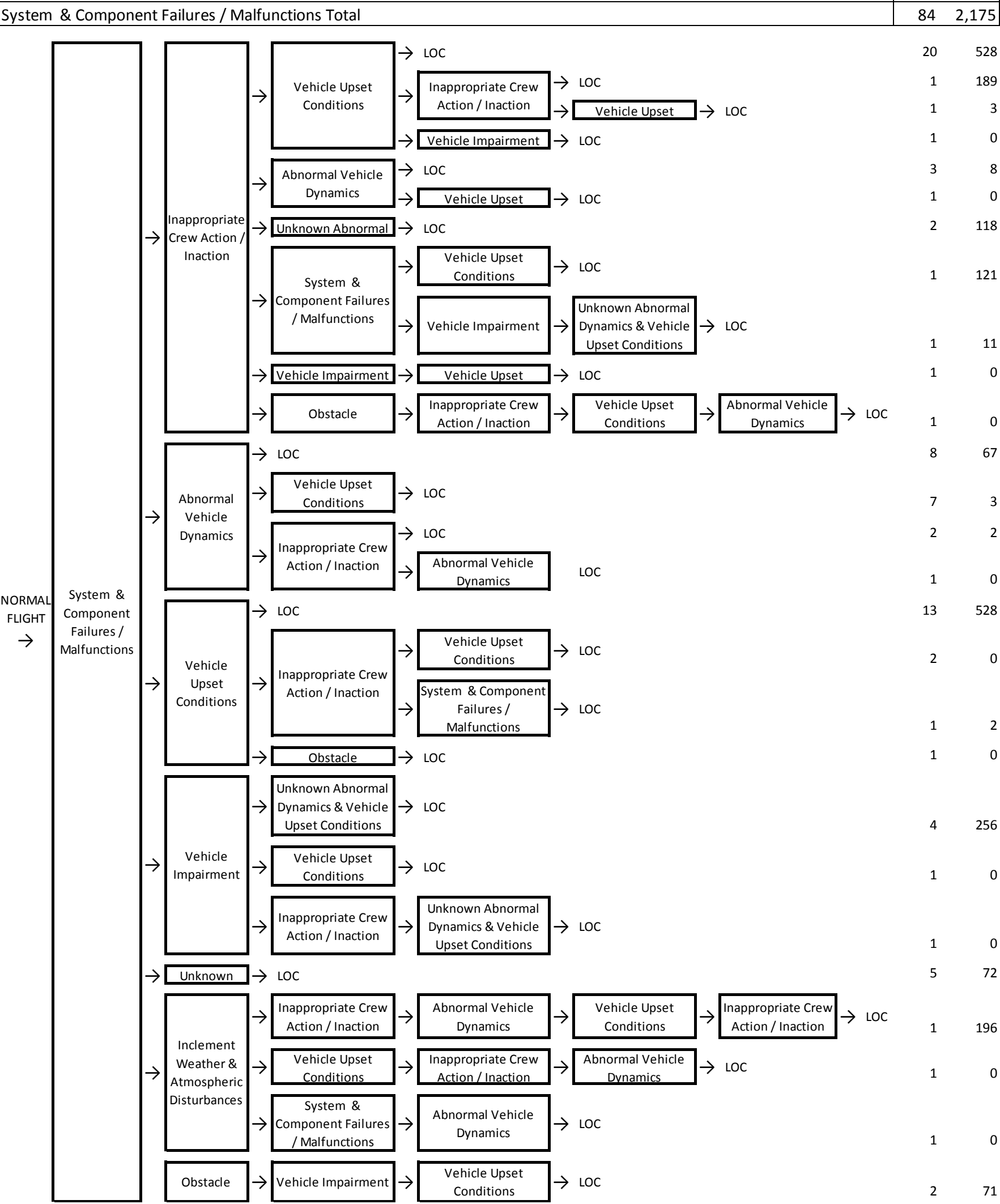

Figure B-1. LOC Sequences Initiated by System \& Component Failures / Malfunctions.

26

American Institute of Aeronautics and Astronautics 
Events Fatalities

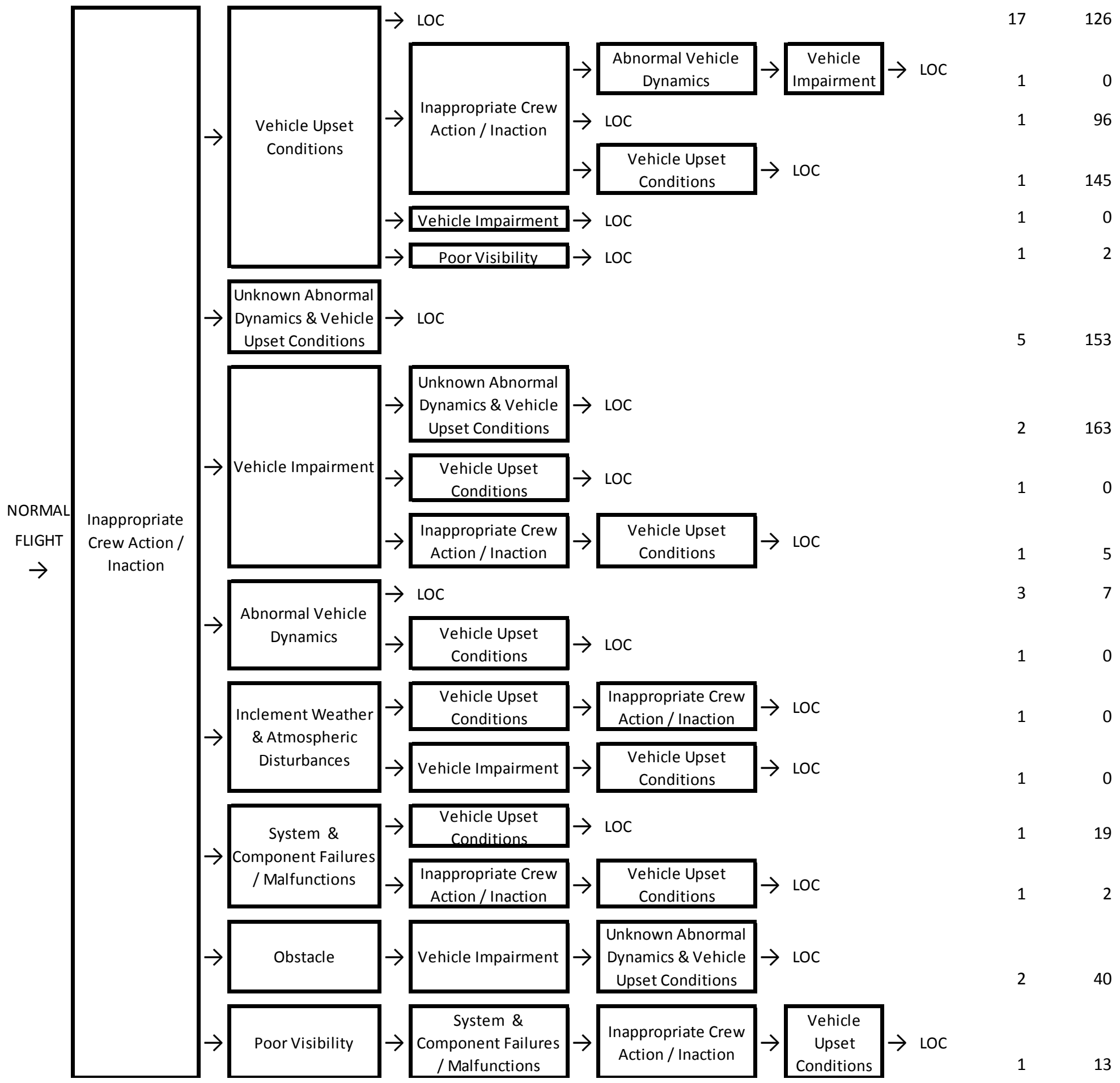

Figure B-2. LOC Sequences Initiated by Inappropriate Crew Action / Inaction. 


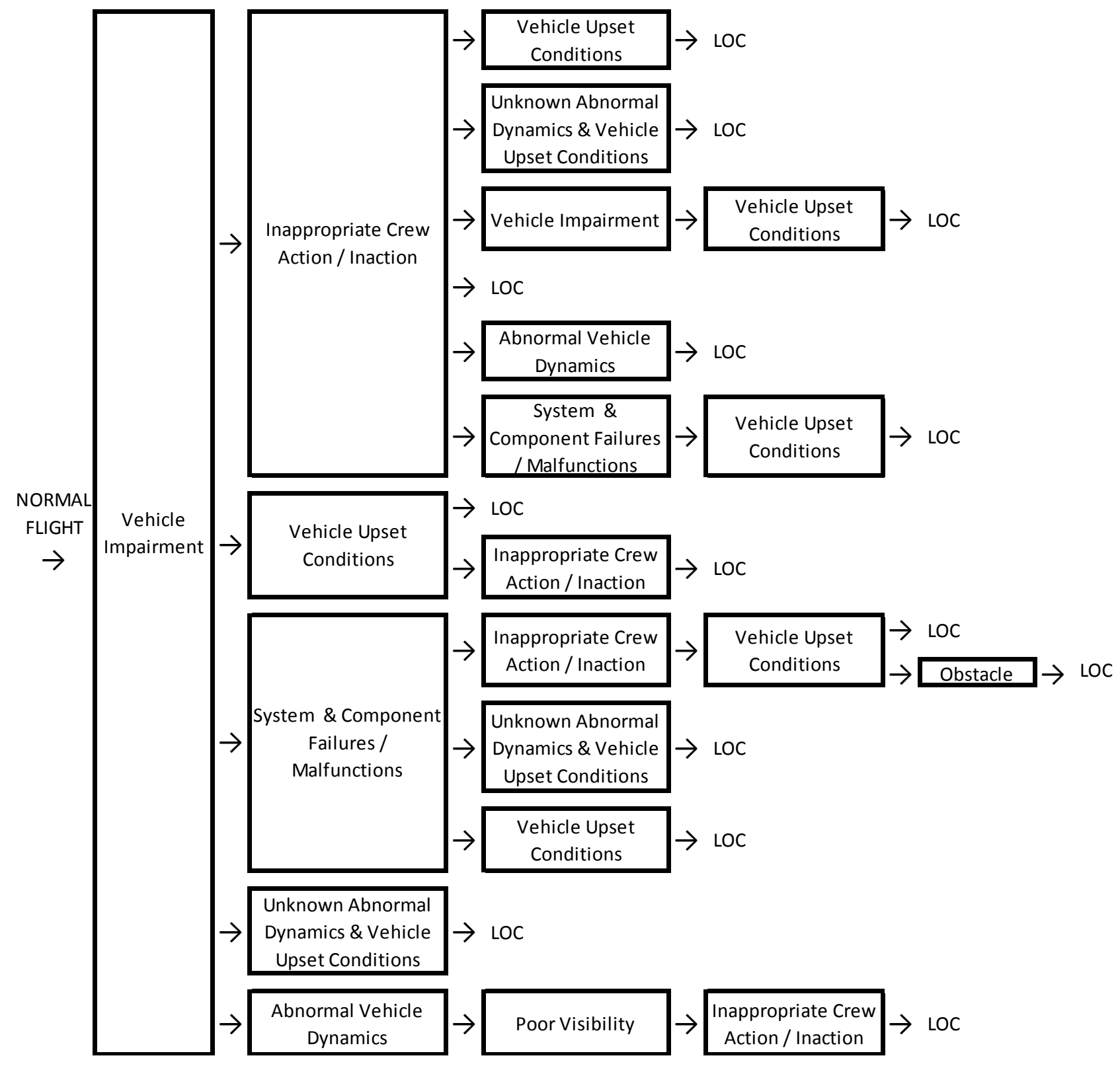

5

5

2

1

1

1

Figure B-3. LOC Sequences Initiated by Vehicle Impairment. 


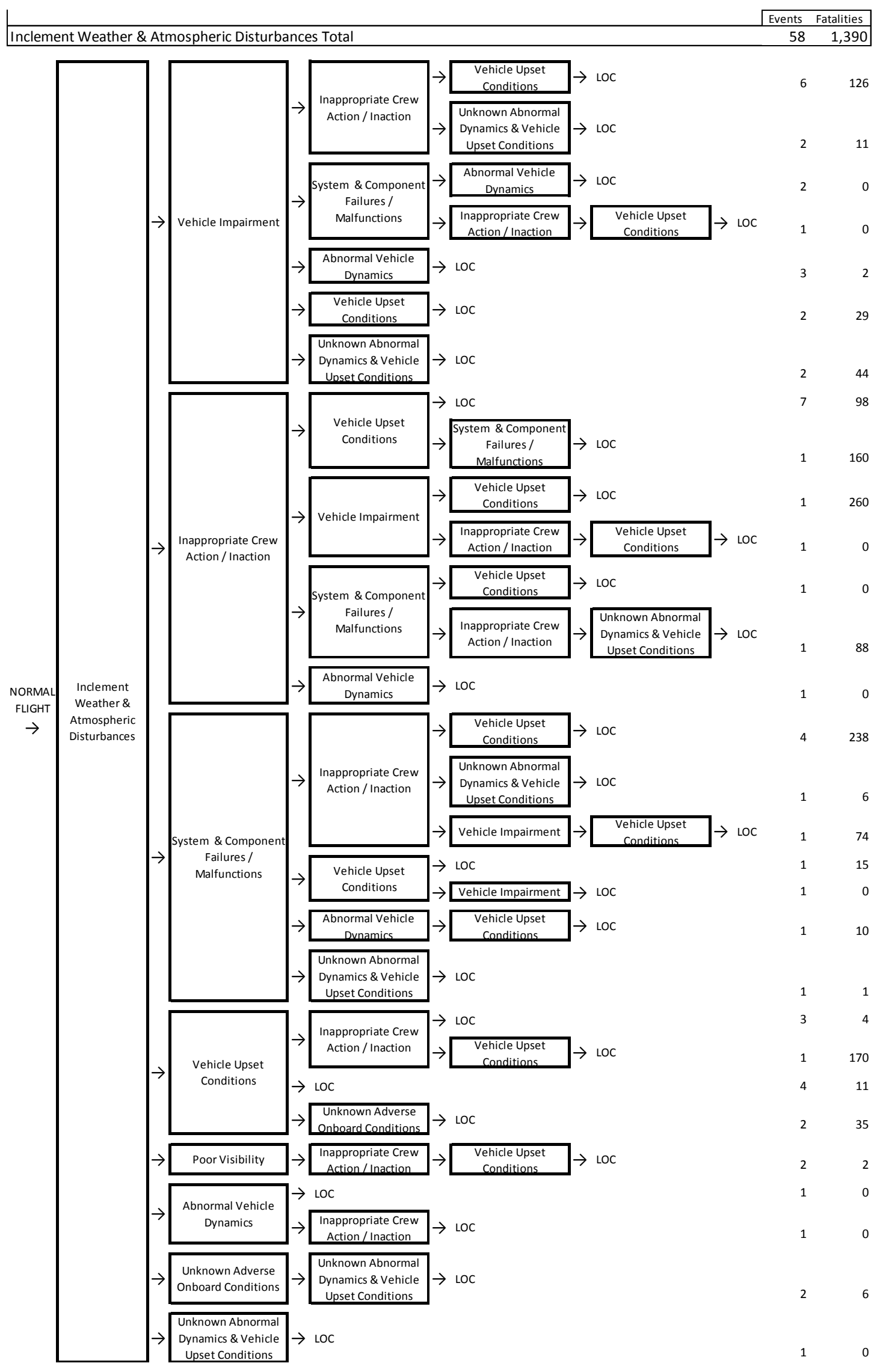

Figure B-4. LOC Sequences Initiated by Inclement Weather \& Atmospheric Disturbances.

29

American Institute of Aeronautics and Astronautics 


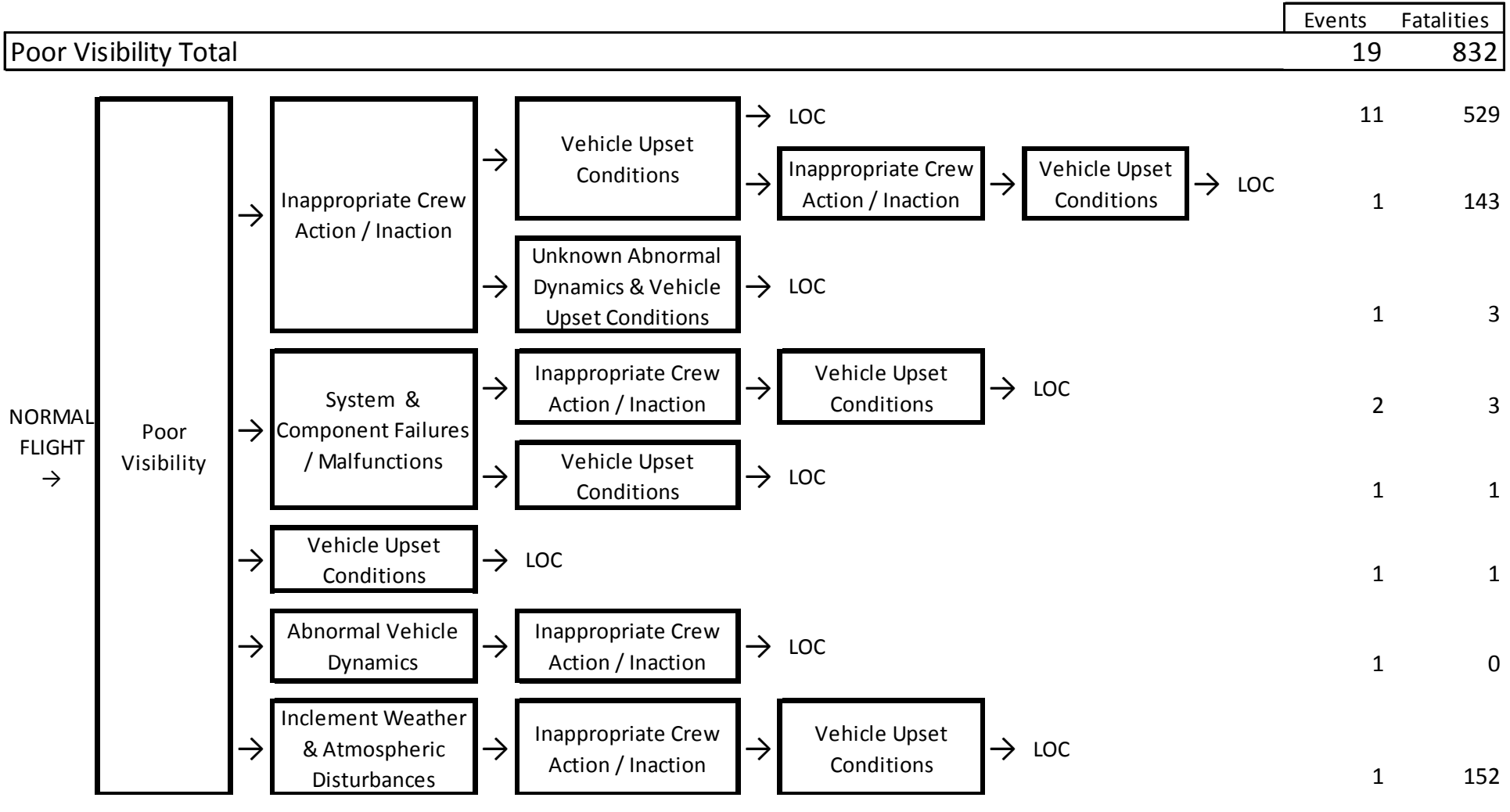

Figure B-5. LOC Sequences Initiated by Poor Visibility.

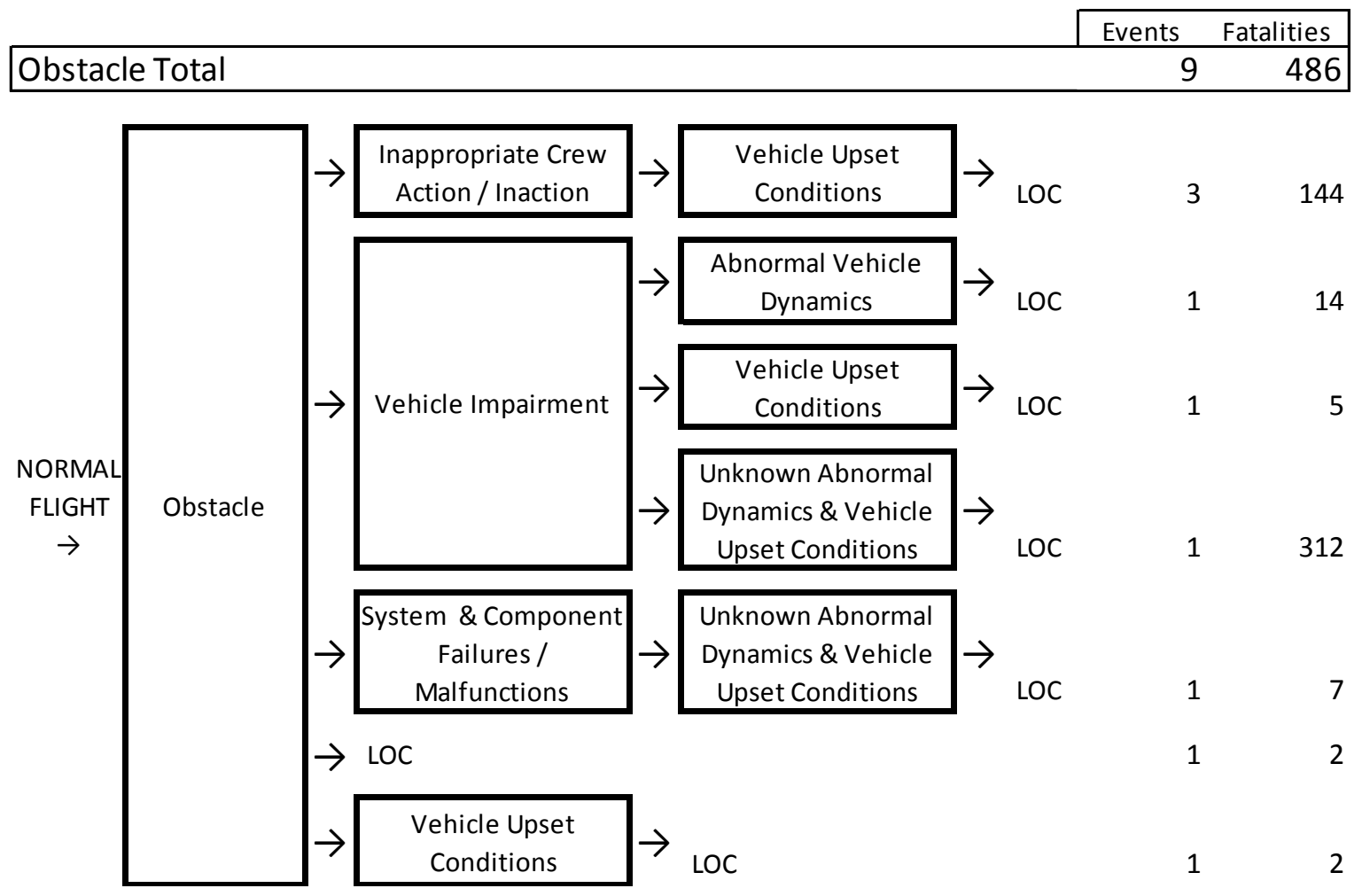

Figure B-6. LOC Sequences Initiated by an Obstacle.

American Institute of Aeronautics and Astronautics 


\section{Acknowledgments}

The first author would like to thank Dr. Liang Tang of United Technologies Corporation (UTC) for the introduction on generating three-dimensional scatter plots using Matlab ${ }^{\mathrm{TM}}$, and Dr. Bart Bacon of NASA Langley for the information he provided on using Matlab ${ }^{\mathrm{TM}}$ to read data from Excel files. This support provided the basis for analyzing worst-case precursor combinations in terms of number of accidents and fatalities.

\section{Dedication}

The research presented in this paper is dedicated to the memory and research contributions of Dr. Celeste M. Belcastro of NASA Langley Research Center, who lost her courageous and selfless battle with cancer and passed from this life on August 22, 2008. She dedicated her life and career to aviation safety research, and made numerous technical and leadership contributions in the areas of vehicle health management and safety-critical avionics systems. Just prior to her illness, she had embarked on a research collaboration with her identical twin, Dr. Christine M. Belcastro, to address aircraft loss of control. Her absence from this work will forever be a significant and irreparable loss to the aerospace research community. 


\section{References}

1 “Statistical Summary of Commercial Jet Airplane Accidents, Worldwide Operations, 1959-2012”, Boeing Commercial Airplanes, July 2013. URL: http://www.boeing.com/news/techissues/pdf/statsum.pdf

2 Evans, Joni K., “An Examination of In Flight Loss of Control Events During 1988-2004,” Alliant Techsystems, Inc., NASA Langley Research Center, Contract No.: TEAMS:NNL07AM99T/R1C0, Task No. 5.2, 2007.

3 “Global Fatal Accident Review 1997-2006,” CAP 776, Civil Aviation Authority, July 2008.

URL: http://www.caa.co.uk/docs/33/CAP776.pdf

${ }^{4}$ Wilborn, J. E. and Foster, J. V., "Defining Commercial Aircraft Loss-of-Control: a Quantitative Approach,” AIAA Atmospheric Flight mechanics Conference and Exhibit, AIAA, Providence, Rhode Island, 16-19 August 2004.

${ }^{5}$ Lambregts, A. A., Nesemeier, G., Wilborn, J. E., and Newman, R. L., "Airplane Upsets: Old Problem, New Issues,” AIAA Modeling and Simulation Technologies Conference and Exhibit, 2008, AIAA 2008-6867.

${ }^{6}$ Belcastro, Christine M. and Foster, John V., “Aircraft Loss-of-Control Accident Analysis,” AIAA Guidance, Navigation and Control Conference, Toronto, 2010.

7 Newman, Richard L., “Thirty Years of Airline Loss-of-Control Mishaps,” AIAA Guidance, Navigation and Control Conference, Minneapolis, Minnesota, 2012.

${ }^{8}$ Belcastro, Christine M. and Jacobson, Steven, "Future Integrated Systems Concept for Preventing Aircraft Lossof-Control Accidents," AIAA Guidance, Navigation and Control Conference, Toronto, 2-5 August 2010.

9 Belcastro, Christine M., "Loss of Control Prevention and Recovery: Onboard Guidance, Control, and Systems Technologies,” AIAA Guidance, Navigation and Control Conference, Minneapolis, Minnesota, 2012.

10 Belcastro, Christine M., "Validation and Verification of Future Integrated Safety-Critical Systems Operating under Off-Nominal Conditions,” AIAA Guidance, Navigation and Control Conference, Toronto, 2010.

${ }^{11}$ Belcastro, Christine M., Validation \& Verification of Safety-Critical Systems Operating under Off-Nominal Conditions. Chapter 20 of the Book Entitled: Optimization-Based Clearance of Flight Control Laws, Springer, 2011.

${ }^{12}$ Belcastro, Christine M.: Validation of Safety-Critical Systems for Aircraft Loss-of-Control Prevention and Recovery, AIAA Guidance, Navigation and Control Conference, Minneapolis, Minnesota, 2012.

13 R. Dorsett, Aircraft Accident Reports on DVD, Flight Simulation Systems, Austin, TX, 2006. 